\title{
OPTIMAL SWITCHING BETWEEN TWO RANDOM WALKS
}

\author{
By R. CAiroli and Robert C. Dalang ${ }^{1}$
}

\section{Ecole Polytechnique Fédérale and Tufts University}

\begin{abstract}
This paper is motivated by remarkable results of Mandelbaum, Shepp and Vanderbei concerning an optimal switching problem for two Brownian motions. In this paper, the discrete form of this problem, in which the Brownian motions are replaced by random walks, is studied and solved without any restriction on the boundary data. The method proposed here involves uncovering the structure of the solution using combinatorial and geometric arguments, and then providing a characterization for the two types of possible solutions, as well as explicit formulas for computing the solution. The extension of these methods and results to the continuous time problem will be considered in a subsequent paper.
\end{abstract}

1. Introduction. This paper arose from an attempt at understanding some remarkable recent work of Mandelbaum [8] and Mandelbaum, Shepp and Vanderbei [9]. These authors studied and solved a particular optimal switching problem under certain regularity assumptions on the boundary data. The problem is an interesting and highly nontrivial generalization of the classical optimal stopping problem for a single Brownian motion on an interval [2] and can be described as follows.

Consider two independent Brownian motions $X^{1}=\left(X_{t^{1}}^{1}, t^{1} \in \mathbb{R}_{+}\right)$and $X^{2}=\left(X_{t^{2}}^{2}, t^{2} \in \mathbb{R}_{+}\right)$killed at the endpoints of the intervals $\left[0, N^{1}\right]$ and $\left[0, N^{2}\right]$, respectively, where $N^{1}$ and $N^{2}$ are positive numbers. Imagine that an observer can control the evolution of $X^{1}$ and $X^{2}$ separately, that is, can leave $t^{1}$ fixed and let $t^{2}$ increase or leave $t^{2}$ fixed and let $t^{1}$ increase. This determines a process $\left(\left(X_{t^{1}}^{1}, X_{t^{2}}^{2}\right),\left(t^{1}, t^{2}\right) \in \mathbb{R}_{+}^{2}\right)$ which evolves in $D=\left[0, N^{1}\right] \times\left[0, N^{2}\right]$. Assume that the observer can choose the switching strategy and the time at which the evolution ends, knowing that at that time he will receive a reward which only depends on the state of the process. This reward is represented by a nonnegative real-valued payoff function $f$ defined on the boundary $D$, and the objective is to maximize the expected reward.

This problem fits into the well-developed theory of stochastic control [3], [5] and [7] which tells us that the solution goes through the determination of the value function. The basic result of the general theory is that the value function satisfies the so-called Hamilton-Jacobi-Bellman equation, which in this case is a second order nonlinear partial differential equation. Since the smoothness of $v$ is not known in advance, it is already nontrivial to give a meaning to the equation and to decide in which space one should prove ex-

Received April 1994; revised November 1994.

${ }^{1}$ Research partially supported by NSF Grant DMS-91-03962 and ARO Grants DAAL-03-92-60323 and DAAH-04-94-G-0261.

AMS 1991 subject classifications. 60G40, 62L15, 49L25.

Key words and phrases. Stochastic control, optimal switching, random walk, value function.

1982 
istence and uniqueness, but this is now handled by the recently developed theory of viscosity solutions. Analytic aspects of this particular problem were studied by Evans and Friedman [4].

Of course, writing the equation and proving existence of the value function does not give much insight into the nature of the solution or of the optimal control. In this particular problem, which has a very special form, the objective is to determine the optimal solution explicitly. The solution in [9] relied on the so-called principle of smooth fit, which suggests that the expected reward can be found by solving certain differential equations. The authors carried out this resolution, which involves a significant amount of calculations and a discussion of several cases. It turns out that the behavior of the optimally controlled process is particularly intricate and involves a complicated switching phenomenon along certain curves, which can be described by a Brownian local time.

While the direct calculations in [9] do lead to the solution (under regularity assumptions), they do not explain why the solution has the remarkable structure obtained, and while this problem presents many interesting technical difficulties related to the fact that it is a continuous-time problem, the essential features are already captured by its discrete analogue, in which the Brownian motions are replaced by random walks. This discrete problem (which is in fact equivalent to the continuous problem with piecewise linear data) has the advantage that its statement is completely elementary. On the other hand, for discrete control problems, the only available tool is to write the dynamic programming equation, which is typically equivalent to a problem of optimization under constraints that can only be solved numerically. From this point of view, the discrete problem might be considered harder than the original problem, in which tools from differential equations such as the principle of smooth fit are available.

In this paper, we solve the discrete form of the problem above, with the Brownian motions replaced by random walks. Since the state space is now finite and no differential equations can be written, we rely on significantly different methods from those of [9]. We characterize the value function as the biconcave envelope of the payoff function (see Proposition 2.1), and then use a purely combinatorial and elementary (though intricate) argument to determine the structure of the solution essentially without computations and, in particular, without first computing the value function. The solutions can be of two types, according to whether or not there is a central subrectangle of $D$ where all directions of motion are optimal (see Theorem 3.5). In this indifference region, the biconcave envelope of the payoff function is actually bilinear; that is, its graph is a hyperbolic paraboloid.

Somewhat surprisingly, it turns out that this qualitative study can be completed by an explicit determination of the optimal switching strategy in terms of the payoff function. Given the structural results above, the main problem reduces to determining certain switching curves. This is achieved in Theorem 4.2 by using a geometric argument. Conditions are then provided on the boundary data for the solution to be either of the two possible types (Theorem 4.4) 
and provide an explicit solution valid in essentially all cases (Theorems 4.6 and 4.7).

A geometric interpretation of the solution can be given as follows. In each square of an integer grid in $D$, the graph of the value function can be thought of as a hyperbolic paraboloid. These hyperbolic paraboloids are pieced together in such a way that the value function is biconcave. This is similar to constructing a concave function by piecing together straight line segments. In the original continuous-time problem, the integer grid must conceptually be replaced by an infinitesimal grid.

This continuous-time extension will be considered in a future paper. We also anticipate that the methods developed here will be useful in solving the three-dimensional version of this problem, which to date has only been solved for very special forms of the payoff function [11]. It should also be mentioned that it is an open problem to solve the optimal switching problem when the Brownian motions evolve in any domain other than a rectangle (with boundary segments parallel to the directions of motion), such as a triangle.

The main results of this paper were announced in [1].

2. Stating the problem. Fix two integers $N^{1}, N^{2} \geq 2$. For $m \leq n$, the interval $\{m, \ldots, n\}$ of $\mathbb{N}$ will be denoted $[m, n]$, and when $m \leq n-2,] m, n[$ denotes the interval $[m+1, n-1]$.

Let $(\Omega, \mathscr{F}, P)$ be a complete probability space. For $i=1,2$, let $X^{i}=$ $\left(X_{t^{i}}^{i}, t^{i} \in \mathbb{N}\right)$ be a simple random walk on $\left[0, N^{i}\right]$ with absorption at the endpoints; in particular, given $X_{t^{i}}^{i}=m$, where $0<m<N^{i}, X_{t^{i}+1}^{i}$ equals $m+1$ or $m-1$ with probability $1 / 2$ each. We assume that $X^{1}$ and $X^{2}$ are independent.

Imagine that an observer can control the evolution of $X^{1}$ and $X^{2}$ separately, that is, can leave $t^{1}$ fixed and let $t^{2}$ increase or leave $t^{2}$ fixed and let $t^{1}$ increase. This determines a process $X=\left(\left(X_{t^{1}}^{1}, X_{t^{2}}^{2}\right),\left(t^{1}, t^{2}\right) \in \mathbb{N}^{2}\right)$ which evolves in $D=$ $\left[0, N^{1}\right] \times\left[0, N^{2}\right]$ that we term a switched process. As in the continuous-time problem described in the Introduction, we assume that the observer can choose the switching strategy and the time at which the evolution ends, knowing that at that time he will receive a reward which only depends on the state of the process. This reward is represented by a nonnegative real-valued payoff function $f$ defined on $D$ which vanishes in the interior $\left.D^{\circ}=\right] 0, N^{1}[\times] 0, N^{2}[$ of $D$, and the objective is to maximize the expected reward.

Notice that once the switched process $\left(X_{t}\right)$ reaches one of the four boundary segments of $D$, it cannot leave this segment. So from that time on, the problem reduces to the classical optimal stopping problem for a single random walk absorbed at the endpoints of an interval. The solution to this classical problem [2] involves computing the concave envelope of the payoff function (i.e., the smallest concave function larger than the payoff function) and stopping the random walk the first time the random walk visits a state where the concave envelope is equal to the payoff function. It is therefore convenient to eliminate this last phase of the evolution, which can be achieved simply by assuming 
that $f$ is concave on each boundary segment of $D$, that is,

$$
f(\cdot, 0), f\left(N^{1}, \cdot\right), f\left(\cdot, N^{2}\right) \text { and } f(0, \cdot) \text { are concave functions. }
$$

Under this assumption, the evolution of the switched process terminates at the time of the first visit of $X$ to $\partial D=D \backslash D^{\circ}$, and therefore the problem is to determine a switching strategy that maximizes the expected reward.

To formulate this problem precisely, let $\mathscr{F}_{t^{i}}^{i}=\sigma\left(X_{s^{i}}^{i}, 0 \leq s^{i} \leq t^{i}\right), i=1,2$. For $x=\left(x^{1}, x^{2}\right)$, the conditional distribution of $X$ given $X_{(0,0)}=x$ will be denoted $P_{x}$ with corresponding expectation operator $E_{x}$. For $t=\left(t^{1}, t^{2}\right) \in \mathbb{N}^{2}$, we set $\mathscr{F}_{t}=\mathscr{F}_{t^{1}}^{1} \vee \mathscr{F}_{t^{2}}^{2}$. The family $\left(\mathscr{F}_{t}, t \in \mathbb{N}^{2}\right)$ is then a filtration indexed by $\mathbb{N}^{2}$ (cf. [10], Section 2), that is, $\mathscr{F}_{0}$ is complete and $\mathscr{F}_{s^{1}, s^{2}} \subset \mathscr{F}_{t^{1}, t^{2}}$ if $s^{1} \leq t^{1}$ and $s^{2} \leq t^{2}$.

For $t=\left(t^{1}, t^{2}\right) \in \mathbb{N}^{2}$, let $|t|=t^{1}+t^{2}$. A predictable increasing path (p.i.p.) (or switching strategy) is a family $\left(Z_{n}, n \in \mathbb{N}\right)$ of random variables with values in $\mathbb{N}^{2}$ such that $Z_{0}=0, Z_{n}^{i} \leq Z_{n+1}^{i}$ for $i=1,2,\left|Z_{n+1}-Z_{n}\right|=1$ and, for all $s$ and $t,\left\{Z_{n}=s, Z_{n+1}=t\right\} \in \mathscr{F}_{s}$, for all $n \in \mathbb{N}$. Observe that $\left|Z_{n}\right|=n$ and that, for $i=1,2, Z_{n}^{i}$ represents the number of times that we have let $X^{i}$ evolve up to time $n$. The switched process is now the process $X^{Z}=\left(X_{Z_{n}}, n \in \mathbb{N}\right)$, and we often write $X_{n}^{Z}$ instead of $X_{Z_{n}}$. This process evolves in the rectangle $D$.

Given a p.i.p. $Z$, we set

$$
\tau^{Z}=\inf \left\{n \in \mathbb{N}: X_{n}^{Z} \in \partial D\right\}
$$

Assume that a nonnegative real-valued function $f$ defined on $\partial D$ is given. As mentioned above, there is no loss of generality in assuming that the restriction of $f$ to each segment of $\partial D$ is concave, $i=1, \ldots, 4$. The optimal switching problem is now to find a p.i.p. $Z^{*}$ such that

$$
E_{x}\left(f\left(X_{\tau^{Z^{*}}}^{Z^{*}}\right)\right)=g(x), \quad \text { where } g(x)=\sup _{Z} E_{x}\left(f\left(X_{\tau^{Z}}^{Z}\right)\right)
$$

and the supremum is over all p.i.p.'s $Z$. As will become apparent, the key to solving this problem is understanding the structure of the function $g$. For this, we need some definitions.

A real-valued function $h$ defined on $D$ is $v$-concave (resp. $h$-concave) if $h\left(x^{1}, \cdot\right)$ [resp. $\left.h\left(\cdot, x^{2}\right)\right]$ is concave for all $x^{1} \in\left[0, N^{1}\right]$ (resp. for all $x^{2} \in\left[0, N^{2}\right]$ ). Recall that a real-valued function $\phi$ defined on an interval $[m, n]$ of $\mathbb{N}$ is concave if and only if, for each $k \in] m, n$ [,

$$
\frac{1}{2} \phi(k-1)+\frac{1}{2} \phi(k+1) \leq \phi(k) .
$$

A $v$-linear or h-linear function is defined by replacing "concave" by "linear" above and the inequalities by equalities in (2) "linear" as defined here is sometimes termed "affine" in the literature). A real-valued function $h$ defined on $D$ is biconcave if it is both $v$-concave and $h$-concave. Such a function is bilinear if it is both $v$-linear and $h$-linear.

We say that $h: D \rightarrow \mathbb{R}$ is a biconcave majorant of $f$ if $h$ is biconcave in $D$ and $h(x) \geq f(x)$ for all $x \in \partial D$. When the set of biconcave majorants of $f$ has a smallest element, we term this element the biconcave envelope of $f$. 
Proposition 2.1. (a) The function $g$ is the biconcave envelope of $f$.

(b) Let

$$
\begin{aligned}
& \mathscr{E}_{1}=\left\{x \in D^{\circ}: \frac{1}{2} g\left(x^{1}-1, x^{2}\right)+\frac{1}{2} g\left(x^{1}+1, x^{2}\right)=g\left(x^{1}, x^{2}\right)\right\}, \\
& \mathscr{E}_{2}=\left\{x \in D^{\circ}: \frac{1}{2} g\left(x^{1}, x^{2}-1\right)+\frac{1}{2} g\left(x^{1}, x^{2}+1\right)=g\left(x^{1}, x^{2}\right)\right\} .
\end{aligned}
$$

Then $\mathscr{E}_{1} \cup \mathscr{E}_{2}=D^{\circ}$, and an optimal switching strategy is to leave $t^{2}$ fixed and let $X^{1}$ evolve if $\left(X_{t^{1}}^{1}, X_{t^{2}}^{2}\right) \in \mathscr{E}_{1}$ and to leave $t^{1}$ fixed and let $X^{2}$ evolve if $\left(X_{t^{1}}^{1}, X_{t^{2}}^{2}\right) \in \mathscr{E}_{2}$.

PROOF. This proposition is a special case of a theorem of Mandelbaum and Vanderbei. According to [10], Theorem 3, $g$ is the smallest multiexcessive majorant of the function $\tilde{f}$ defined on $D$ which coincides with $f$ on $\partial D$ and which vanishes in $D^{\circ}$. In the terminology of [10] adapted to the present context, a function $h: D \rightarrow \mathbb{R}$ is multiexcessive if

$$
h\left(x^{1}, x^{2}\right) \geq \frac{1}{2} h\left(x^{1}-1, x^{2}\right)+\frac{1}{2} h\left(x^{1}+1, x^{2}\right)
$$

and

$$
h\left(x^{1}, x^{2}\right) \geq \frac{1}{2} h\left(x^{1}, x^{2}-1\right)+\frac{1}{2} h\left(x^{1}, x^{2}+1\right) .
$$

Therefore statement (a) follows from comparing the definitions.

Statement (b) is equivalent to the description of the optimal strategy provided in [10], Section 3.1.

The main objective of this paper is now to describe the sets $\mathscr{E}_{1}$ and $\mathscr{E}_{2}$ explicitly. Their structure is described in the next section, and in Section 4.3 we will show how these sets are determined by the payoff function $f$.

3. The structure of the solution. We are going to determine the structure of the solution to the optimal switching problem without first evaluating the biconcave envelope $g$. For this, it is necessary to introduce some definitions and to make a few crucial observations.

3.1. Propagation of linearity. Given two points $a=\left(a^{1}, a^{2}\right)$ and $b=\left(b^{1}, b^{2}\right)$ of $D$ such that $a^{1} \leq b^{1}$ and $a^{2} \leq b^{2}$, the rectangle $\left[a^{1}, b^{1}\right] \times\left[a^{2}, b^{2}\right]$ will also be denoted $[a, b]$. If $a^{2}=b^{2}$ (resp. $a^{1}=b^{1}$ ), this rectangle is degenerate and is a horizontal (resp. vertical) segment. The notions of boundary $\partial[a, b]$ and interior $[a, b]^{\circ}$ are defined in the same way as for the rectangle $D$ itself.

A subset $F$ of $D$ is $h$-convex (resp. $v$-convex) if it contains all horizontal (resp. vertical) segments $[x, y]$ whose extremities $x$ and $y$ belong to $F$; a subset is biconvex if it is both $h$-convex and $v$-convex.

A real-valued function $\tilde{f}$ defined on $D$ is $h$-concave on an $h$-convex subset $F$ of $D$ if the function $\tilde{f}\left(\cdot, x^{2}\right)$ is concave on $\left[x^{1}, y^{1}\right]$ for each horizontal segment $\left[x^{1}, y^{1}\right] \times\left\{x^{2}\right\} \subset F$. One defines similarly the properties " $\tilde{f}$ is $h$-linear on $F$ " and " $\tilde{f}$ is $v$-concave or $v$-linear on a $v$-convex subset $F$ of $D$." The function $f$ is bilinear on a biconvex subset $F$ of $E$ if it is both $h$-linear and $v$-linear 
on $F$. Moreover, we will say that $\tilde{f}$ is strictly $h$-concave at a point $\left(x^{1}, x^{2}\right) \in$ ] $0, N^{1}\left[\times\left[0, N^{2}\right]\right.$ if

$$
\frac{1}{2} \tilde{f}\left(x^{1}-1, x^{2}\right)+\frac{1}{2} \tilde{f}\left(x^{1}+1, x^{2}\right)<\tilde{f}\left(x^{1}, x^{2}\right)
$$

and strictly $v$-concave at a point $\left.\left(x^{1}, x^{2}\right) \in\left[0, N^{1}\right] \times\right] 0, N^{2}[$ if

$$
\frac{1}{2} \tilde{f}\left(x^{1}, x^{2}-1\right)+\frac{1}{2} \tilde{f}\left(x^{1}, x^{2}+1\right)<\tilde{f}\left(x^{1}, x^{2}\right) .
$$

The following proposition establishes a property of "propagation of linearity and of strict concavity" that will be the key to the structure of the solution to the optimal switching problem.

Proposition 3.1. Let $[a, b] \subset D$ be a rectangle with nonempty interior.

(a) If $g$ is linear on the horizontal (resp. vertical) boundary segments of $[a, b]$, then $g$ is h-linear (resp. v-linear) in $[a, b]$.

(b) If in addition, $g$ is strictly $v$-concave (resp. h-concave) at some point $\left(a^{1}, x^{2}\right)$ or $\left(b^{1}, x^{2}\right)$ for some $\left.x^{2} \in\right] a^{2}, b^{2}\left[\right.$ [resp. $\left(x^{1}, a^{2}\right)$ or $\left(x^{1}, b^{2}\right)$ for some $\left.x^{1} \in\right] a^{1}, b^{1}[]$, then $g$ is strictly $v$-concave (resp. h-concave) at $\left(x^{1}, x^{2}\right)$ for all $\left.x^{1} \in\right] a^{1}, b^{1}\left[\right.$ [resp. for all $\left.x^{2} \in\right] a^{2}, b^{2}[]$.

PROOF. (a) We only consider the case where $g$ is linear on the horizontal boundary segments of $[a, b]$, as the other case is similar. Let $g_{1}$ be the function defined on $D$ by

$$
g_{1}\left(x^{1}, x^{2}\right)= \begin{cases}\alpha\left(x^{1}\right) g\left(a^{1}, x^{2}\right)+\beta\left(x^{1}\right) g\left(b^{1}, x^{2}\right), & \text { if }\left(x^{1}, x^{2}\right) \in[a, b]^{\circ}, \\ g\left(x^{1}, x^{2}\right), & \text { otherwise, }\end{cases}
$$

where $\alpha\left(x^{1}\right)=\left(b^{1}-x^{1}\right) /\left(b^{1}-a^{1}\right)$ and $\beta\left(x^{1}\right)=\left(x^{1}-a^{1}\right) /\left(b^{1}-a^{1}\right)$. Then $g_{1} \leq g$ since $g$ is $h$-concave. Moreover, $g_{1}$ is clearly $h$-concave. We now prove that $g_{1}$ is $v$-concave. Since $g_{1}$ coincides with $g$ outside $[a, b]^{\circ}, g_{1}\left(x^{1}, \cdot\right)$ is concave for $\left.x^{1} \notin\right] a^{1}, b^{1}\left[\right.$. If $\left.x^{1} \in\right] a^{1}, b^{1}\left[\right.$, then $g_{1}\left(x^{1}, \cdot\right)$ is $v$-concave at $x^{2}$ if $x^{2} \notin\left[a^{2}, b^{2}\right]$. If $\left.x^{2} \in\right] a^{2}, b^{2}\left[\right.$, then since $g\left(a^{1}, \cdot\right)$ and $g\left(b^{1}, \cdot\right)$ are concave,

$$
\begin{aligned}
\frac{1}{2}\left(g_{1}(\right. & \left.\left.x^{1}, x^{2}-1\right)+g_{1}\left(x^{1}, x^{2}+1\right)\right) \\
= & \alpha\left(x^{1}\right) \frac{1}{2}\left(g\left(a^{1}, x^{2}-1\right)+g\left(a^{1}, x^{2}+1\right)\right) \\
& +\beta\left(x^{1}\right) \frac{1}{2}\left(g\left(b^{1}, x^{2}-1\right)+g\left(b^{1}, x^{2}+1\right)\right) \\
\leq & \alpha\left(x^{1}\right) g\left(a^{1}, x^{2}\right)+\beta\left(x^{1}\right) g\left(b^{1}, x^{2}\right) \\
= & g_{1}\left(x^{1}, x^{2}\right) .
\end{aligned}
$$

Therefore $g_{1}\left(x^{1}, \cdot\right)$ is concave at $x^{2}$. If $x^{2} \in\left\{a^{2}, b^{2}\right\}$, then definition (2) and the fact that $g_{1} \leq g$ imply that $g_{1}\left(x^{1}, \cdot\right)$ is concave at $x^{2}$. Therefore, $g_{1}$ is a biconcave majorant of $f$ and so $g_{1} \geq g$. We have shown that $g_{1}=g$ and the conclusion in (a) follows.

(b) Assume, for example, that in addition $g\left(a^{1},.\right)$ is strictly $v$-concave at $\left(a^{1}, x^{2}\right)$ for some $\left.x^{2} \in\right] a^{2}, b^{2}\left[\right.$. Then for $\left.x^{1} \in\right] a^{1}, b^{1}[$ the inequality in (4) becomes strict, so $g$ is strictly $v$-concave at $\left(x^{1}, x^{2}\right)$. 
REMARK 3.2. (a) Let $[a, b]$ be as in Proposition 3.1. If $g$ is linear on the horizontal boundary segments of $[a, b]$, then for all $\left(x^{1}, x^{2}\right) \in[a, b]$,

$$
g\left(x^{1}, x^{2}\right)=\frac{b^{1}-x^{1}}{b^{1}-a^{1}} g\left(a^{1}, x^{2}\right)+\frac{x^{1}-a^{1}}{b^{1}-a^{1}} g\left(b^{1}, x^{2}\right) .
$$

Similarly, if $g$ is linear on the vertical boundary segments of $[a, b]$, then for all $\left(x^{1}, x^{2}\right) \in[a, b]$,

$$
g\left(x^{1}, x^{2}\right)=\frac{b^{2}-x^{2}}{b^{2}-a^{2}} g\left(x^{1}, a^{2}\right)+\frac{x^{2}-a^{2}}{b^{2}-a^{2}} g\left(x^{1}, b^{2}\right) .
$$

If $g$ is linear on all four boundary segments of $[a, b]$, then $g$ is bilinear in $[a, b]$. Replacing $g\left(a^{1}, x^{2}\right)$ and $g\left(b^{1}, x^{2}\right)$ in (5) by the expression furnished in (6), we get (after rearranging the terms)

$$
\begin{aligned}
g\left(x^{1}, x^{2}\right)= & g\left(a^{1}, a^{2}\right)+\frac{x^{1}-a^{1}}{b^{1}-a^{1}}\left(g\left(b^{1}, a^{2}\right)-g\left(a^{1}, a^{2}\right)\right) \\
& +\frac{x^{2}-a^{2}}{b^{2}-a^{2}}\left(g\left(a^{1}, b^{2}\right)-g\left(a^{1}, a^{2}\right)\right) \\
& +\frac{\left(x^{1}-a^{1}\right)\left(x^{2}-a^{2}\right)}{\left(b^{1}-a^{1}\right)\left(b^{2}-a^{2}\right)}\left(g\left(b^{1}, b^{2}\right)-g\left(b^{1}, a^{2}\right)\right. \\
& \left.-g\left(a^{1}, b^{2}\right)+g\left(a^{1}, a^{2}\right)\right) .
\end{aligned}
$$

This formula shows that the graph of $g$ on $[a, b]$ is the restriction to $[a, b]$ of the hyperbolic paraboloid generated by the quadrilateral of $\mathbb{R}^{3}$ with vertices given in the canonical coordinate system $O e^{1} e^{2} e^{3}$ by

$$
\begin{array}{ll}
A_{1}=\left(a^{1}, a^{2}, g\left(a^{1}, a^{2}\right)\right), & A_{2}=\left(b^{1}, a^{2}, g\left(b^{1}, a^{2}\right)\right), \\
A_{3}=\left(b^{1}, b^{2}, g\left(b^{1}, b^{2}\right)\right), & A_{4}=\left(a^{1}, b^{2}, g\left(a^{1}, b^{2}\right)\right),
\end{array}
$$

that is, the restriction of the quadric surface generated by lines parallel to the plane $O e^{1} e^{2}$ which meet the line segments $A_{1} A_{4}$ and $A_{2} A_{3}$ (this surface is also generated by the lines parallel to the plane $O e^{2} e^{3}$ which meet the line segments $A_{1} A_{2}$ and $A_{3} A_{4}$ ).

(b) Assume $a^{1}<b^{1}$ and $a^{2}<b^{2}$. Given a quadruple $\left(c_{1}, c_{2}, c_{3}, c_{4}\right)$ of real numbers, there is exactly one bilinear function $g$ defined on $[a, b]$ such that

$$
g\left(a^{1}, a^{2}\right)=c_{1}, \quad g\left(b^{1}, a^{2}\right)=c_{2}, \quad g\left(b^{1}, b^{2}\right)=c_{3}, \quad g\left(a^{1}, b^{2}\right)=c_{4} .
$$

The values of $g$ in $[\dot{a}, b]$ are given by (7). Notice that if $\tilde{g}$ is the bilinear function associated to the quadruple $\left(\tilde{c}_{1}, c_{2}, c_{3}, c_{4}\right)$, then

$$
c_{1} \leq \tilde{c}_{1} \Leftrightarrow g\left(x^{1}, x^{2}\right) \leq \tilde{g}\left(x^{1}, x^{2}\right) \text { for all }\left(x^{1}, x^{2}\right) \in\left[a^{1}, b^{1}\left[\times\left[a^{2}, b^{2}[.\right.\right.\right.
$$

This equivalence remains true if $\leq$ is replaced by $<,>$ or $\geq$. 
3.2. Vertical and horizontal convexity. The conclusion of Proposition 3.1 leads to important geometric properties of $\mathscr{E}_{1}$ and $\mathscr{E}_{2}$.

PROPOSITION 3.3. $\mathscr{E}_{1}$ is v-convex and $\mathscr{E}_{2}$ is $h$-convex.

PROOF. Let $x$ and $y$ be two points in $\mathscr{E}_{1}$ such that $x^{1}=y^{1}$ and $y^{2}-x^{2}>1$. By the definition of $\mathscr{E}_{1}, g$ is $h$-linear on the horizontal boundary segments of the rectangle $\left[\left(x^{1}-1, x^{2}\right),\left(x^{1}+1, y^{2}\right)\right]$. By Proposition 3.1, we deduce that $g$ is $h$-linear on this rectangle. Again by the definition of $\mathscr{E}_{1}$, the vertical segment $[x, y]$ is therefore contained in $\mathscr{E}_{1}$. It follows that $\mathscr{E}_{1}$ is $v$-convex. The proof that $\mathscr{E}_{2}$ is $h$-convex is similar.

We will say that a function $\xi$ defined on $\left[0, N^{2}\right]$ with values in $\left[0, N^{1}\right]$ is unimodal of type 1 (resp. unimodal of type 2) if there exists $n \in\left[0, N^{2}\right]$ such that $\xi$ is nondecreasing (resp. nonincreasing) on $[0, n]$ and nonincreasing (resp. nondecreasing) on $\left[n, N^{2}\right]$.

The following proposition establishes the existence of two particularly important unimodal functions.

Proposition 3.4. Assume that one of the two following hypotheses is satisfied:

(a) There exists $\left.x^{1} \in\right] 0, N^{1}\left[\right.$ such that $\left(x^{1}, x^{2}\right) \in \mathscr{E}_{2}$ for all $\left.x^{2} \in\right] 0, N^{2}[$.

(b) For all $\left.x^{2} \in\right] 0, N^{2}\left[\right.$, there exists $\left.x^{1} \in\right] 0, N^{1}\left[\right.$ such that $\left(x^{1}, x^{2}\right) \in \mathscr{E}_{2} \backslash \mathscr{E}_{1}$.

Then there exist two unimodal functions $\xi_{1}$ and $\xi_{2}$, respectively of type 1 and type 2 , such that $\xi_{1}(0)=\xi_{1}\left(N^{2}\right)=0, \xi_{2}(0)=\xi_{2}\left(N^{2}\right)=N^{1}$ and $0<\xi_{1}(n) \leq$ $\xi_{2}(n)<N^{1}$ for all $\left.n \in\right] 0, N^{2}[$, which give rise to the inclusions

$$
F \subset \mathscr{E}_{1} \text { and } D^{\circ} \backslash F \subset \mathscr{E}_{2} \text {, }
$$

where $F$ denotes the set $\left\{\left(x^{1}, x^{2}\right) \in D^{\circ}: x^{1}<\xi_{1}\left(x^{2}\right)\right.$ or $\left.x^{1}>\xi_{2}\left(x^{2}\right)\right\}$.

Proof. For all $n \in] 0, N^{2}[$, set

$$
\begin{aligned}
& \mu_{1}(n)= \begin{cases}\inf \left\{m \in\left[0, N^{1}\right]:(m, n) \in \mathscr{E}_{2}\right\}, & \text { if } n \in] 0, N^{2}[, \\
0, & \text { if } n \in\left\{0, N^{2}\right\},\end{cases} \\
& \mu_{2}(n)= \begin{cases}\sup \left\{m \in\left[0, N^{1}\right]:(m, n) \in \mathscr{E}_{2}\right\}, & \text { if } n \in] 0, N^{2}[, \\
N^{1}, & \text { if } n \in\left\{0, N^{2}\right\} .\end{cases}
\end{aligned}
$$

Notice that $0<\mu_{1}(n) \leq \mu_{2}(n)<N^{1}$ for all $\left.n \in\right] 0, N^{2}[$. Moreover, set

$$
p^{1}=\sup _{n \in\left[0, N^{2}\right]} \mu_{1}(n) \quad \text { and } \quad q^{1}=\inf _{n \in\left[0, N^{2}\right]} \mu_{2}(n) \text {. }
$$


Clearly, $0<p^{1}<N^{1}$ and $0<q^{1}<N^{1}$. Choose $p^{2}$ and $q^{2}$ in $] 0, N^{2}[$ such that

$$
\mu_{1}\left(p^{2}\right)=p^{1} \text { and } \mu_{2}\left(q^{2}\right)=q^{1} .
$$

Let $\xi_{1}$ and $\xi_{2}$ denote the two functions defined, respectively, on $\left[0, N^{2}\right]$ by

$$
\begin{gathered}
\xi_{1}(n)= \begin{cases}\sup _{k \in[0, n]} \mu_{1}(k), & \text { if } n \in\left[0, p^{2}\right], \\
\sup _{k \in\left[n, N^{2}\right]} \mu_{1}(k), & \text { if } n \in\left[p^{2}, N^{2}\right],\end{cases} \\
\xi_{2}(n)= \begin{cases}\inf _{k \in[0, n]} \mu_{2}(k), & \text { if } n \in\left[0, q^{2}\right], \\
\inf _{k \in\left[n, N^{2}\right]} \mu_{2}(k), & \text { if } n \in\left[q^{2}, N^{2}\right] .\end{cases}
\end{gathered}
$$

Clearly, $\xi_{1}$ and $\xi_{2}$ are two unimodal functions, respectively of type 1 and type 2, independent of the choice of $p^{2}$ and $q^{2}$ and such that $\xi_{1}(0)=\xi_{1}\left(N^{2}\right)=0$, $\xi_{2}(0)=\xi_{2}\left(N^{2}\right)=N^{1}, 0<\mu_{1}(n) \leq \xi_{1}(n)$ and $\xi_{2}(n) \leq \mu_{2}(n)<N^{1}$ for all $n \in] 0, N^{2}[$. Moreover, these two functions satisfy

$$
\begin{gathered}
\xi_{1}\left(p^{2}\right)=\sup _{n \in\left[0, N^{2}\right]} \xi_{1}(n)=\mu_{1}\left(p^{2}\right)=p^{1}, \\
\xi_{2}\left(q^{2}\right)=\inf _{n \in\left[0, N^{2}\right]} \xi_{2}(n)=\mu_{2}\left(q^{2}\right)=q^{1} .
\end{gathered}
$$

Define $F$ as in the statement of the proposition and let us prove that the inclusions in (8) hold. By Proposition 3.3, $\mathscr{E}_{2}$ is $h$-convex; therefore, $\mathscr{E}_{2}=$ $\left\{\left(x^{1}, x^{2}\right) \in D^{\circ}: \mu_{1}\left(x^{2}\right) \leq x^{1} \leq \mu_{2}\left(x^{2}\right)\right\}$. Consequently, $D^{\circ} \backslash F=\left\{\left(x^{1}, x^{2}\right) \in\right.$ $\left.D^{\circ}: \xi_{1}\left(x^{2}\right) \leq x^{1} \leq \xi_{2}\left(x^{2}\right)\right\} \subset \mathscr{E}_{2}$, which establishes the second inclusion in (8). In order to establish the first inclusion, it suffices by Proposition 2.1(b) and (9) to show that if $\left(x^{1}, x^{2}\right) \in D^{\circ}$ is such that $\mu_{1}\left(x^{2}\right) \leq x^{1}<\xi_{1}\left(x^{2}\right)$ or $\xi_{2}\left(x^{2}\right)<x^{1} \leq$ $\mu_{2}\left(x^{2}\right)$, then $\left(x^{1}, x^{2}\right)$ belongs to $\mathscr{E}_{1}$. Assume that the first alternative holds, since the proof in the other case is analogous. Since $\mu_{1}\left(p^{2}\right)=\xi_{1}\left(p^{2}\right)$, we conclude that $x^{2} \neq p^{2}$. Assume, for example, that $x^{2}<p^{2}$. Then there exists $n \in] 0, x^{2}\left[\right.$ such that $\xi_{1}\left(x^{2}\right)=\xi_{1}(n)=\mu_{1}(n)$. However, $x^{1}<\xi_{1}\left(x^{2}\right)=\mu_{1}(n)$ and $x^{1}<\xi_{1}\left(x^{2}\right) \leq \xi_{1}\left(p^{2}\right)=\mu_{1}\left(p^{2}\right)$; therefore, $\left(x^{1}, n\right)$ and $\left(x^{1}, p^{2}\right)$ belong to $\mathscr{E}_{1}$. By Proposition $3.3,\left(x^{1}, x^{2}\right)$ also belongs to $\mathscr{E}_{1}$.

It remains to prove that $\xi_{1} \leq \xi_{2}$. Assume that hypothesis (a) is satisfied. Then $\mu_{1}\left(x^{2}\right) \leq x^{1} \leq \mu_{2}\left(x^{2}\right)$ for all $\left.x^{2} \in\right] 0, N^{2}\left[\right.$ and, therefore, $\xi_{1} \leq \xi_{2}$. Assume now that hypothesis (b) is satisfied. If there exists $\left.x^{2} \in\right] 0, N^{2}[$ such that $\xi_{1}\left(x^{2}\right)>\xi_{2}\left(x^{2}\right)$, then $\left(x^{1}, x^{2}\right)$ belongs to $F$, therefore to $\mathscr{E}_{1}$, for all $\left.x^{1} \in\right] 0, N^{1}[$, which contradicts the hypothesis; consequently, $\xi_{1} \leq \xi_{2}$.

3.3. The two types of structures. Denote the optimal switching problem by $(\pi)$. In order to explain the structure of the solution of $(\pi)$, it is convenient to be able to replace $(\pi)$ by an equivalent problem $(\tilde{\pi})$ obtained by symmetry. Denote by $\Phi_{1}$ and $\Phi_{2}$, respectively, the reflection with respect to the vertical line $x^{1}=N^{1} / 2$ and the reflection with respect to the first diagonal, that is, the line whose equation is $x^{2}=x^{1}$. Let us also denote by $\tilde{D}, \tilde{E}_{1}$ and $\tilde{\mathscr{E}}_{2}$ the 
respective images of $D, \mathscr{E}_{1}$ and $\mathscr{E}_{2}$ (resp. $D, \mathscr{E}_{2}$ and $\mathscr{E}_{1}$ ) under $\Phi_{1}$ (resp. $\Phi_{2}$ ). Obviously $\tilde{D}=D$ if $i=1$ and $\tilde{D}=\left[0, N^{2}\right] \times\left[0, N^{1}\right]$ if $i=2$. Let $(\tilde{\pi})$ denote the optimal switching problem relative to the function $\tilde{f}=f \circ \Phi_{i}$ on $\partial \tilde{D}$. It is easy to check that $\tilde{g}=g \circ \Phi_{i}$ is the biconcave envelope of $\tilde{f}$ and that $\tilde{E}_{1}$ and $\tilde{\mathscr{E}}_{2}$ are the regions where the optimal directions for $(\tilde{\pi})$ are, respectively, horizontal and vertical. It follows that the structure of the solution of $(\pi)$ can be deduced from the structure of the solution of $(\tilde{\pi})$, and conversely.

The results that we are about to formulate rely on the conclusions of Proposition 3.4. It is therefore necessary to make sure that one of the two hypotheses of this proposition is satisfied. If hypothesis (b) is not satisfied, that is, there exists $\left.x^{2} \in\right] 0, N^{2}\left[\operatorname{such}\right.$ that $\left(x^{1}, x^{2}\right) \in \mathscr{E}_{1}$ for all $\left.x^{1} \in\right] 0, N^{1}[$, then it suffices to transform $(\pi)$ by applying $\Phi_{2}$ to see that $\left(x^{2}, y^{2}\right) \in \tilde{E}_{2}$ for all $\left.y^{2} \in\right] 0, N^{1}[$ and therefore that hypothesis (a) is satisfied for the transformed problem. After possibly transforming $(\pi)$ by applying $\Phi_{2}$, we can therefore assume that one of the two hypotheses of Proposition 3.4 is satisfied and therefore that the conclusions of this proposition apply. In particular, we will make use of the integers $p^{1}, q^{1}, p^{2}$ and $q^{2}$ defined in (10) and (11).

The inclusions in (8) can be expressed by saying that the horizontal direction is optimal in $F$ and that the vertical direction is optimal in $D^{\circ} \backslash F$. Of course, it is possible that in a portion of $F$ or of $D^{\circ} \backslash F$, both directions are optimal. In the case where $p^{1} \leq q^{1}$, there is nothing to add to what has just been explained. On the other hand, in the case where $p^{1}>q^{1}$, we are going to show that there exists an indifference rectangle around which are four regions in which the horizontal and vertical directions are alternately optimal (cf. Figure 1; the arrows there represent the optimal direction of motion in each region).

In the sequel, we will say that the solution is of type 1 (resp. type 2) if after possibly having transformed $(\pi)$ by applying $\Phi_{2}$, one of the two assumptions of Proposition 3.4 is satisfied and the inequality $p^{1} \leq q^{1}$ (resp. $p^{1}>q^{1}$ ) holds.

The structure of the solution of type 2 will be described in the following theorem. Notice that this type of solution is only possible in the case where $N^{1}>2$ and $N^{2}>2$. Moreover, since $p^{1}>q^{1}$, it is clear that $p^{2} \neq q^{2}$. In order
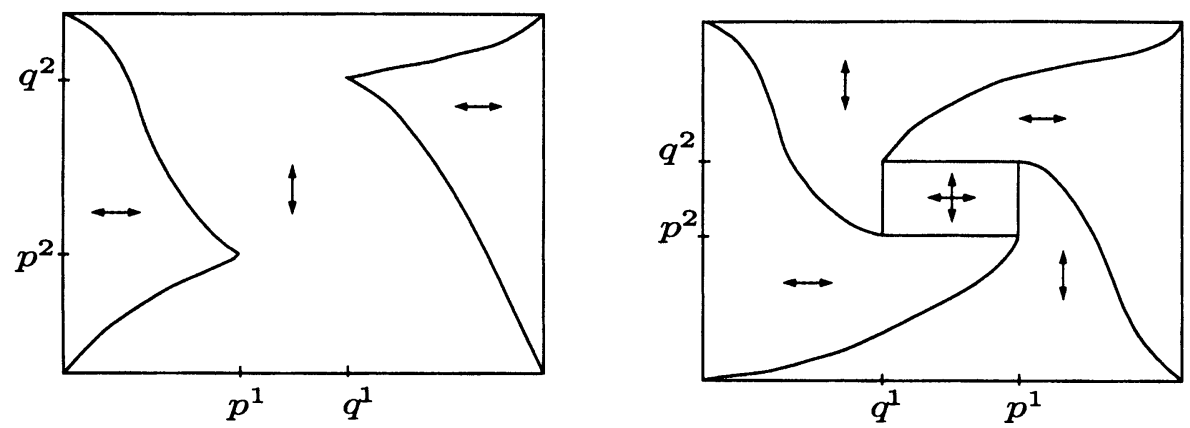

FIG. 1. Solutions of type 1 (left) and type 2 (right). 
to avoid dealing with two cases, we will assume that $p^{2}<q^{2}$. If this condition is not satisfied, then it will be after applying the transformation $\Phi_{1}$.

THEOREM 3.5. Assume that one of the two hypotheses of Proposition 3.4 is satisfied, and define $p^{1}, q^{1}, p^{2}$ and $q^{2}$ by (10) and (11), with $p^{2}$ and $q^{2}$ chosen such that the value $\left|p^{2}-q^{2}\right|$ is as small as possible. Moreover, define $\xi_{1}$ and $\xi_{2}$ by (12) and (13). If $p^{1}>q^{1}$ and $p^{2}<q^{2}$, then

$$
\left.\xi_{1}(n) \leq q^{1} \quad \text { for all } n \in\right] p^{2}, N^{2}\left[, \quad \xi_{2}(n) \geq p^{1} \quad \text { for all } n \in\right] 0, q^{2}[.
$$

Moreover, the structure of the solution to the optimal switching problem is described by the following conclusions: The horizontal direction is optimal in $F_{1}$ and $F_{3}$, where

$$
\begin{aligned}
& F_{1}=\left\{\left(x^{1}, x^{2}\right) \in D^{\circ}: x^{1}<\xi_{1}\left(x^{2}\right)\right\}, \\
& F_{3}=\left\{\left(x^{1}, x^{2}\right) \in D^{\circ}: x^{1}>\xi_{2}\left(x^{2}\right)\right\},
\end{aligned}
$$

the vertical direction is optimal in $F_{2}$ and $F_{4}$, where

$$
\begin{aligned}
& F_{2}=\left\{\left(x^{1}, x^{2}\right) \in D^{\circ}: x^{2}<p^{2} \text { and } \xi_{1}\left(x^{2}\right) \leq x^{1} \leq \xi_{2}\left(x^{2}\right)\right. \\
& \text { or } \left.p^{2} \leq x^{2}<q^{2} \text { and } p^{1} \leq x^{1} \leq \xi_{2}\left(x^{2}\right)\right\} \\
& F_{4}=\left\{\left(x^{1}, x^{2}\right) \in D^{\circ}: p^{2}<x^{2}<q^{2} \text { and } \xi_{1}\left(x^{2}\right) \leq x^{1} \leq q^{1}\right. \\
& \text { or } \left.x^{2}>q^{2} \text { and } \xi_{1}\left(x^{2}\right) \leq \overline{x^{1}} \leq \bar{\xi}_{2}\left(x^{2}\right)\right\}
\end{aligned}
$$

and both directions are optimal in the (possibly empty) rectangle $] q^{1}, p^{1}[\times$ ]$p^{2}, q^{2}[$.

Proof. By hypothesis, the conclusions of Proposition 3.4 apply. By this proposition, the segments $\left[q^{1}, p^{1}\left[\times\left\{p^{2}\right\}\right.\right.$ and $\left.] q^{1}, p^{1}\right] \times\left\{q^{2}\right\}$ are both contained in $\mathscr{E}_{1}$. As $\mathscr{E}_{1}$ is $v$-convex by Proposition 3.3 , it follows that $] q^{1}, p^{1}\left[\times\left[p^{2}, q^{2}\right] \subset\right.$ $\mathscr{E}_{1}$.

We now prove (15). Since $\xi_{1}(n) \leq \xi_{1}\left(q^{2}\right) \leq \xi_{2}\left(q^{2}\right)=q^{1}$ for all $n \in\left[q^{2}, N^{2}\right]$ and $\xi_{2}(n) \geq \xi_{2}\left(p^{2}\right) \geq \xi_{1}\left(p^{2}\right)=p^{1}$ for all $n \in\left[0, p^{2}\right]$, it is sufficient to prove that $\xi_{1}(n) \leq q^{1}$ and $\xi_{2}(n) \geq p^{1}$ for all $\left.n \in\right] p^{2}, q^{2}\left[\right.$. If $q^{2}-p^{2}=1$, there is nothing to prove. Assume that $q^{2}-p^{2}>1$. If $p^{1}-q^{1}=1$, then $\xi_{1}(n) \leq q_{1}$ for all $n \in] p^{2}, q^{2}$, since $\xi_{1}(n)<p^{1}$ for all $\left.n \in\right] p^{2}, q^{2}\left[\right.$ because $q^{2}-p^{2}$ is minimal. Similarly, we see that $\xi_{2}(n) \geq p^{1}$ for all $n \in$ ] $p^{2}, q^{2}$ [. If $p^{1}-q^{1}>1$, assume that $\xi_{1}(n)>q^{1}$ for some $\left.n \in\right] p^{2}, q^{2}$. Since $\xi_{1}\left(q^{2}\right) \leq \xi_{2}\left(q^{2}\right)=q^{1}$, there exists $k \in] p^{2}, q^{2}\left[\right.$ such that $\xi_{1}(k)=\mu_{1}(k)>q^{1}$. Consequently $\left(q^{1}, k\right) \notin \mathscr{E}_{2}$, in other words, $g$ is strictly $v$-concave at $\left(q^{1}, k\right)$. Taking into account the fact that $\xi_{1}(k)<p^{1}$ due to the minimal character of $q^{2}-p^{2}$, by applying Proposition $3.1(b)$ to the rectangle $\left[q^{1}, p^{1}\right] \times[k-1, k+1]$, we deduce that $\left(\xi_{1}(k), k\right) \notin \mathscr{E}_{2}$. This contradicts the second inclusion in (8). We therefore conclude that $\xi_{1}(n) \leq$ $q^{1}$ for all $\left.n \in\right] p^{2}, q^{2}$ [. Similarly, we see that $\xi_{2}(n) \geq p^{1}$ for all $\left.n \in\right] p^{2}, q^{2}[$. Assertion (15) is therefore established. The final conclusions of the theorem follow directly from (8) and from the inclusion $] q^{1}, p^{1}[\times] p^{2}, q^{2}\left[\subset \mathscr{E}_{1} \cap \mathscr{E}_{2}\right.$. 
REMARK 3.6. It is now possible to describe the evolution of the optimally switched process. Fix $\left.x^{2} \in\right] 0, p^{2}\left[\right.$, assume that $\xi_{1}\left(x^{2}\right)<p^{1}$ and let $y^{2}=$ $\left.\left.\inf \{n \in] x^{2}, p^{2}\right]: \xi_{1}(n)>\xi_{1}\left(x^{2}\right)\right\}$. If $\left.z^{2} \in\right] 0, y^{2}\left[\right.$, then on the set $\left\{X_{0}=\right.$ $\left.\left(\xi_{1}\left(x^{2}\right), z^{2}\right)\right\}$, the optimal strategy is as follows. Initially, leave $t^{1}$ fixed and equal to 0 and let $t^{2}$ evolve until either $X$ visits $\left(\xi_{1}, 0\right)$, in which case the evolution stops and the payoff is $f\left(\xi_{1}\left(x^{2}\right), 0\right)$, or $X$ visits $\left(\xi_{1}\left(x^{2}\right), y^{2}\right)$. In this case, leave $t^{2}$ fixed and let $t^{1}$ evolve until either $X$ visits $\left(0, y^{2}\right)$, in which case the evolution stops and the payoff is $f\left(0, y^{2}\right)$, or $X$ visits $\left(\xi_{1}\left(y^{2}\right), y^{2}\right)$. In this case, proceed as above as though the starting point were $\left(\xi_{1}\left(y^{2}\right), y^{2}\right)$. If $X$ ever visits $\left(p^{1}, p^{2}\right)$, then the optimal strategy depends on whether the solution is of type 1 or 2 .

If the solution is of type 1 , then from this time on leave $t^{1}$ fixed and let $t^{2}$ evolve until $X$ visits $\left(p^{1}, 0\right)$ or $\left(p^{1}, N^{2}\right)$, at which time the evolution terminates and the payoff is, respectively, $f\left(p^{1}, 0\right)$ or $f\left(p^{1}, N^{2}\right)$.

If the solution is of type 2 and $p^{2}<q^{2}$, then leave $t^{1}$ fixed and let $t^{2}$ evolve until either $X$ visits $\left(p^{1}, 0\right)$, at which time the evolution terminates and the payoff is $f\left(p^{1}, 0\right)$, or $X$ visits $\left(p^{1}, q^{2}\right)$. In this case, leave $t^{2}$ fixed and let $t^{1}$ evolve until either $X$ visits $\left(N^{1}, q^{2}\right)$, at which time the evolution terminates and the payoff is $f\left(N^{1}, q^{2}\right)$, or $X$ visits $\left(q^{1}, q^{2}\right)$. The direction of evolution switches each time $X$ visits one of the four states $\left(q^{1}, q^{2}\right),\left(q^{1}, p^{2}\right),\left(p^{1}, p^{2}\right)$ or $\left(p^{1}, q^{2}\right)$, until $X$ finally visits one of the four states $\left(p^{1}, 0\right),\left(N^{1}, q^{2}\right),\left(q^{1}, N^{2}\right)$ or $\left(0, p^{2}\right)$, at which time the evolution terminates and the payoff is $f\left(p^{1}, 0\right)$, $f\left(N^{1}, q^{2}\right), f\left(q^{1}, N^{2}\right)$ or $f\left(0, p^{2}\right)$, respectively.

4. Computing the solution. Theorem 3.5 provides a complete description of the structure of the solution, and our objective is now to show how the solution is determined by the boundary data. This is a two-step process, which involves first determining whether the solution is of type 1 or 2 and finding the integers $p^{1}, q^{1}, p^{2}$ and $q^{2}$ which satisfy (10) and (11), and then providing a formula for the functions $\xi_{1}$ and $\xi_{2}$ defined in (12) and (13). We shall proceed in reverse order; that is, we assume in this section that the values of $p^{1}, q^{1}$, $p^{2}$ and $q^{2}$ are given and determine a formula for $\xi_{1}$ and $\xi_{2}$. This will provide considerable insight which will be useful in Section 4.3, where we will then provide an explicit and essentially complete solution to the problem.

4.1. Constructing the switching curves. Consider the two functions $\phi^{1}$ and $\phi^{2}$ defined, respectively, on $\left[0, N^{1}\left[\right.\right.$ and $\left[0, N^{2}[\right.$ by

$$
\begin{aligned}
& \phi^{1}\left(x^{1}\right)=\left(x^{1}+1\right) f\left(x^{1}, 0\right)-x^{1} f\left(x^{1}+1,0\right), \\
& \phi^{2}\left(x^{2}\right)=\left(x^{2}+1\right) f\left(0, x^{2}\right)-x^{2} f\left(0, x^{2}+1\right) .
\end{aligned}
$$

Notice that $\phi^{1}(0)=\phi^{2}(0)=f(0,0)$.

REMARK 4.1. (a) The quantity $\phi^{1}\left(x^{1}\right)$ is precisely the value at 0 of the unique linear function $h$ defined on $\left[0, N^{1}\right]$ which coincides with $f(\cdot, 0)$ at $x^{1}$ 
and $x^{1}+1$. As such, $\phi^{1}\left(x^{1}\right)$ can be thought of geometrically as the intercept on the $x^{3}$-axis of $\mathbb{R}^{3}$ of the "line tangent at $x^{1}$ to the graph of $f(\cdot, 0)$." An analogous relationship exists between $\phi^{2}$ and $f(0, \cdot)$.

(b) Since $f(\cdot, 0)$ and $f(0, \cdot)$ are concave, it follows immediately that $\phi^{1}$ and $\phi^{2}$ are nondecreasing.

As in the statement of Theorem 3.5, we assume without loss of generality that the conclusions of Proposition 3.4 are satisfied. The theorem below indicates how to compute the values of $\xi_{1}$ on the interval ]0, $p^{2}$. The values of $\xi_{1}$ on $] p^{2}, N^{2}$ [, or of $\xi_{2}$, can be computed analogously.

THEOREM 4.2. Let $\xi_{1}$ be the function defined in (12). Then, for all $x^{2} \in$ ] $0, p^{2}[$,

$$
\xi_{1}\left(x^{2}\right)= \begin{cases}\left.\left.\inf \left\{x^{1} \in\right] 0, p^{1}\right]: \phi^{1}\left(x^{1}\right) \geq \phi^{2}\left(x^{2}\right)\right\}, & \text { if }\{\} \neq \varnothing, \\ p^{1}, & \text { if }\{\}=\varnothing .\end{cases}
$$

Proof. We assume that $p^{1}>1$; otherwise, there is nothing to prove. It is sufficient to show that

$$
\phi^{1}\left(\xi_{1}\left(x^{2}\right)\right) \geq \phi^{2}\left(x^{2}\right) \quad \text { if } \xi_{1}\left(x^{2}\right)<p^{1},
$$

and

$$
\phi^{1}\left(\xi_{1}\left(x^{2}\right)-1\right)<\phi^{2}\left(x^{2}\right) \text { if } \xi_{1}\left(x^{2}\right)>1 .
$$

Assume that $\xi_{1}\left(x^{2}\right)<p^{1}$ and set

$$
\left.\left.y^{2}=\inf \{n \in] x^{2}, p^{2}\right]: \xi_{1}(n)>\xi_{1}\left(x^{2}\right)\right\} .
$$

Let $h_{1}$ denote the bilinear function on $D$ which coincides with $g$ at each of the four vertices of the rectangle $R=\left[\left(\xi_{1}\left(x^{2}\right), y^{2}-1\right),\left(\xi_{1}\left(x^{2}\right)+1, y^{2}\right)\right]$ and by $h_{2}$ the bilinear function on $D$ whose value at $\left(\xi_{1}\left(x^{2}\right), y^{2}-1\right)$ is

$$
\frac{1}{\xi_{1}\left(x^{2}\right)+1} g\left(0, y^{2}-1\right)+\frac{\xi_{1}\left(x^{2}\right)}{\xi_{1}\left(x^{2}\right)+1} g\left(\xi_{1}\left(x^{2}\right)+1, y^{2}-1\right)
$$

and which coincides with $g$ at the three other vertices of $R$. By Proposition 3.3, the rectangle $\left.\left[\xi_{1}\left(x^{2}\right), \xi_{1}\left(x^{2}\right)+1\right] \times\right] 0, y^{2}$ [is contained in $\mathscr{E}_{2}$; in other words, $g\left(\xi_{1}\left(x^{2}\right), \cdot\right)$ and $g\left(\xi_{1}\left(x^{2}\right)+1, \cdot\right)$ are both linear on $\left[0, y^{2}\right]$. It follows that

$$
h_{1}\left(\xi_{1}\left(x^{2}\right), 0\right)=g\left(\xi_{1}\left(x^{2}\right), 0\right)=f\left(\xi_{1}\left(x^{2}\right), 0\right)
$$

and

$$
h_{1}\left(\xi_{1}\left(x^{2}\right)+1,0\right)=g\left(\xi_{1}\left(x^{2}\right)+1,0\right)=f\left(\xi_{1}\left(x^{2}\right)+1,0\right) .
$$

Similarly, again by Proposition 3.3, $] 0, \xi_{1}\left(y^{2}\right)\left[\times\left\{y^{2}\right\}\right.$ is contained in $\mathscr{E}_{1}$; therefore, $g\left(\cdot, y^{2}\right)$ is linear on $\left[0, \xi_{1}\left(y^{2}\right)\right]$, which implies that $h_{1}, h_{2}, g$ and $f$ coincide at $\left(0, y^{2}\right)$. On the other hand, given the way in which $h_{2}$ is defined, it is easy to see that $h_{2}\left(0, y^{2}-1\right)=g\left(0, y^{2}-1\right)=f\left(0, y^{2}-1\right)$. It follows 
that $h_{1}(0,0)=\phi^{1}\left(\xi_{1}\left(x^{2}\right)\right)$ and $h_{2}(0,0)=\phi^{2}\left(y^{2}-1\right)$. Since $g\left(\cdot, y^{2}-1\right)$ is concave, $h_{1}\left(\xi_{1}\left(x^{2}\right), y^{2}-1\right) \geq h_{2}\left(\xi_{1}\left(x^{2}\right), y^{2}-1\right)$, by (19). Consequently, since $h_{1}$ and $h_{2}$ coincide at the other three vertices of $R$, Remark 3.2(b) implies that $h_{1}(0,0) \geq h_{2}(0,0)$ and, therefore, $\phi^{1}\left(\xi_{1}\left(x^{2}\right)\right) \geq \phi^{2}\left(y^{2}-1\right) \geq \phi^{2}\left(x^{2}\right)$. This proves (17).

We now prove (18). Consider first the case where $x^{2}=1$. In this case, the point $\left(\xi_{1}(1)-1,1\right)$ does not belong to $\mathscr{E}_{2}$ by $(12)$; therefore, $g$ is strictly $v$ concave at this point. Let $h_{1}$ denote the bilinear function on $D$ whose value at $\left(\xi_{1}(1)-1,1\right)$ is

$$
\frac{1}{2} g\left(\xi_{1}(1)-1,0\right)+\frac{1}{2} g\left(\xi_{1}(1)-1,2\right)
$$

and which coincides with $g$ at each of the three other vertices of the rectangle $\left[\left(\xi_{1}(1)-1,1\right),\left(\xi_{1}(1), 2\right)\right]$, and let $h_{2}$ denote the bilinear function which coincides with $g$ at all four vertices of this same rectangle. By Proposition 3.4, $g\left(\xi_{1}(1), \cdot\right)$ is linear on $[0,2]$, whereas $g(\cdot, 1)$ and $g(\cdot, 2)$ are linear on $\left[0, \xi_{1}(1)\right]$, so $h_{1}, h_{2}$ and $f$ coincide at $\left(\xi_{1}(1), 0\right)$ as well as at $(0,2)$. Taking into account the way in which $h_{1}\left(\xi_{1}(1)-1, \cdot\right)$ is defined, we conclude as above that $h_{1}(0,0)=\phi^{1}\left(\xi_{1}(1)-1\right)$ and $h_{2}(0,0)=\phi^{2}(1)$. Given that $g$ is strictly $v$-concave at $\left(\xi_{1}(1)-1,1\right)$, we conclude that $h_{1}\left(\xi_{1}(1)-1,1\right)<h_{2}\left(\xi_{1}(1)-1,1\right)$ and, therefore, that $h_{1}(0,0)<h_{2}(0,0)$ by Remark 3.2(b). It follows that $\phi^{1}\left(\xi_{1}(1)-1\right)<$ $\phi^{2}(1)$, which was to be proved.

Assume now that the statement in (18) is true for $x^{2}=n-1$, where $n \in] 1, p^{2}\left[\right.$. We show that it is also true for $x^{2}=n$. If $\xi_{1}(n-1)<\xi_{1}(n)$, then the point $\left(\xi_{1}(n)-1, n\right)$ does not belong to $\mathscr{E}_{2}$, by (12); therefore, $g$ is strictly $v$-concave at this point. Using the vertices of the rectangle $\left[\left(\xi_{1}(n)-\right.\right.$ $\left.1, n),\left(\xi_{1}(n), n+1\right)\right]$, we conclude that (18) is true, exactly in the same way as when $x^{2}=1$. If $\xi_{1}(n-1)=\xi_{1}(n)$, then the same conclusion follows directly from the hypothesis according to which $\phi^{1}\left(\xi_{1}(n-1)-1\right)<\phi^{2}(n-1)$, using the fact that $\phi^{2}(n-1) \leq \phi^{2}(n)$.

REMARK 4.3. If $\left.\left(x^{1}, x^{2}\right) \in\right] 0, p^{1}[\times] 0, p^{2}[$, then

$$
\begin{array}{lll}
\phi^{1}\left(x^{1}\right) \geq \phi^{2}\left(x^{2}\right) & \text { implies } & \left(x^{1}, x^{2}\right) \in \mathscr{E}_{2}, \\
\phi^{1}\left(x^{1}\right)<\phi^{2}\left(x^{2}\right) & \text { implies } & \left(x^{1}, x^{2}\right) \in \mathscr{E}_{1} .
\end{array}
$$

4.2. Characterizing the type of the solution. In this section, we are going to give a necessary and sufficient condition on the payoff function $f$ for the solution of the optimal switching problem to be of type 1 or of type 2 . This condition is geometric, and will be translated into an analytic condition in Section 4.3 .

Recall that a function $h: D \rightarrow \mathbb{R}$ is a majorant of $f$ if $h(x) \geq f(x)$ for all $x \in \partial D$. If $h$ is a bilinear majorant of $f$, then the contact sets of $h$ with $f$ are the four sets $\mathscr{C}_{1}, \ldots, \mathscr{C}_{4}$ defined as follows: $\mathscr{C}_{1}\left(\right.$ resp. $\left.\mathscr{C}_{3}\right)$ is the set of $\left.x^{1} \in\right] 0, N^{1}[$ such that $h\left(x^{1}, 0\right)=f\left(x^{1}, 0\right)$ [resp. such that $h\left(x^{1}, N^{2}\right)=f\left(x^{1}, N^{2}\right)$ ] and $\mathscr{C}_{2}$ 
(resp. $\left.\mathscr{C}_{4}\right)$ is the set of $\left.x^{2} \in\right] 0, N^{2}\left[\right.$ such that $h\left(N^{1}, x^{2}\right)=f\left(N^{1}, x^{2}\right)$ [resp. such that $h\left(0, x^{2}\right)=f\left(0, x^{2}\right)$. Note that $\mathscr{b}_{1}, \ldots, \mathscr{b}_{4}$ are always intervals and may be singletons or empty. We shall say that this bilinear majorant of $f$ has a type 1 contact with $f$ if

$$
\text { either } \mathscr{b}_{1} \cap \mathscr{C}_{3} \neq \varnothing \text { or } \mathscr{C}_{2} \cap \mathscr{C}_{4} \neq \varnothing \text {, }
$$

and that it has a type 2 contact with $f$ if $\mathscr{C}_{1} \neq \varnothing, \ldots, \mathscr{C}_{4} \neq \varnothing$ and either

$$
\sup \mathscr{b}_{3}<\inf \mathscr{C}_{1} \text { and } \sup \mathscr{b}_{4}<\inf \mathscr{C}_{2}
$$

or

$$
\sup \mathscr{C}_{1}<\inf \mathscr{b}_{3} \quad \text { and } \sup \mathscr{C}_{2}<\inf \mathscr{C}_{4} \text {. }
$$

Notice that the property " $h$ is a bilinear majorant of $f$ with a type 1 (resp. type 2) contact with $f$ " is invariant under the reflections $\Phi_{1}$ and $\Phi_{2}$. Moreover, if $h$ is a bilinear majorant of $f$, then

$$
\left.x^{1} \in \mathscr{C}_{1} \cap \mathscr{C}_{3} \quad \text { implies }\left\{x^{1}\right\} \times\right] 0, N^{2}\left[\subset \mathscr{E}_{2} .\right.
$$

Indeed, if $x^{1} \in \mathscr{C}_{1} \cap \mathscr{C}_{3}$ and if $g$ is the biconcave envelope of $f$, then $g \leq h$ since $h$ is a biconcave majorant of $f$, and $g\left(x^{1}, \cdot\right) \geq h\left(x^{1}, \cdot\right)$ since $g\left(x^{1}, \cdot\right)$ is concave, $h\left(x^{1}, \cdot\right)$ is linear and $g$ and $h$ agree at $\left(x^{1}, 0\right)$ and $\left(x^{1}, N^{2}\right)$. Therefore, $g\left(x^{1}, \cdot\right)=h\left(x^{1}, \cdot\right)$, which proves $(22)$.

THEOREM 4.4. The solution to the optimal switching problem is of type 1 (resp. of type 2) if and only if there exists a bilinear majorant of $f$ which has a type 1 (resp. type 2) contact with $f$.

PROOF. We begin by proving that the condition concerning the type 1 solution is sufficient. Without loss of generality, we suppose that there is a bilinear majorant $h$ of $f$ and $\left.x^{1} \in\right] 0, N^{1}\left[\right.$ such that $h\left(x^{1}, 0\right)=f\left(x^{1}, 0\right)$ and $h\left(x^{1}, N^{2}\right)=f\left(x^{1}, N^{2}\right)$. By (22), hypothesis (a) of Proposition 3.4 is satisfied and $p^{1}=\sup \mu_{1} \leq x^{1} \leq \inf \mu_{2}=p^{2}$, where $\mu_{1}$ and $\mu_{2}$ are defined in (9). The solution is therefore of type 1 .

We now prove that the condition is necessary. Assume the solution is of type 1 and let $g$ be the biconcave envelope of $f$. After possibly transforming the problem by applying $\Phi_{2}$, we can assume that hypothesis (a) of Proposition 3.4 is satisfied.

The case where there exists $x^{1} \in\left[0, N^{1}\left[\right.\right.$ such that both $g\left(x^{1}, \cdot\right)$ and $g\left(x^{1}+\right.$ $1, \cdot)$ are linear on $\left[0, N^{2}\right]$ is quite straightforward. In this case, let $h$ be the bilinear function defined on $D$ that agrees with $g$ at each of the four vertices of the rectangle $\left[x^{1}, x^{1}+1\right] \times\left[0, N^{2}\right]$. Since $g$ is $h$-concave, we conclude that $h \geq g$ on $D$. Therefore, $h$ is a bilinear majorant of $f$ with a type 1 contact with $f$, since either $x^{1}$ or $x^{1}+1$ belongs to $] 0, N^{1}[$.

In the general case, there is $\left.x^{1} \in\right] 0, N^{1}\left[\right.$ such that $g\left(x^{1}, \cdot\right)$ is linear on $\left[0, N^{2}\right]$. Let $k$ be the function defined for $x^{2} \in\left[0, N^{2}\right]$ by

$$
k\left(x^{2}\right)=\frac{1}{2} g\left(x^{1}-1, x^{2}\right)+\frac{1}{2} g\left(x^{1}+1, x^{2}\right) .
$$


Then $k$ is concave and since $g$ is $h$-concave, $g\left(x^{1}, x^{2}\right) \geq k\left(x^{2}\right)$ for all $x^{2} \in$ $\left[0, N^{2}\right]$.

Let $\kappa$ be the linear function defined on $\left[0, N^{2}\right]$ such that $\kappa \geq k$, the function $g\left(x^{1}, \cdot\right)-\kappa(\cdot)$ is constant and $\kappa$ agrees with $k$ at at least one element $x^{2} \in$ $\left[0, N^{2}\right]$.

In the case where $\left.x^{2} \in\right] 0, N^{2}\left[\right.$, define numbers $x_{-}^{3}, x_{+}^{3}, y_{-}^{3}$ and $y_{+}^{3}$ by

$$
x_{ \pm}^{3}=g\left(x^{1} \pm 1, x^{2}-1\right), \quad y_{ \pm}^{3}=2 g\left(x^{1} \pm 1, x^{2}\right)-g\left(x^{1} \pm 1, x^{2}+1\right) .
$$

For $\left(x_{-}, x_{+}\right) \in\left[x_{-}^{3}, y_{-}^{3}\right] \times\left[x_{+}^{3}, y_{+}^{3}\right]$, let $h_{x_{-}, x_{+}}$be the bilinear function defined on $D$ such that

$$
h_{x_{-}, x_{+}}\left(x^{1} \pm 1, x^{2}-1\right)=x_{ \pm}, \quad h_{x_{-}, x_{+}}\left(x^{1} \pm 1, x^{2}\right)=g\left(x^{1} \pm 1, x^{2}\right) .
$$

Since $x_{ \pm} \leq y_{ \pm}^{3}$, observe that

$$
\begin{aligned}
h_{x_{-}, x_{+}}\left(x^{1} \pm 1, x^{2}+1\right) & =2 g\left(x^{1} \pm 1, x^{2}\right)-x_{ \pm} \geq 2 g\left(x^{1} \pm 1, x^{2}\right)-y_{ \pm}^{3} \\
& =g\left(x^{1} \pm 1, x^{2}+1\right),
\end{aligned}
$$

therefore, $h_{x_{-}, x_{+}}\left(x^{1} \pm 1, \cdot\right) \geq g\left(x^{1} \pm 1, \cdot\right)$ since $g\left(x^{1} \pm 1, \cdot\right)$ is $v$-concave. Moreover, using $h$-linearity, we see that

$$
h_{x_{-}^{3}, x_{+}^{3}}\left(x^{1}, x^{2}-1\right)=k\left(x^{2}-1\right) \leq \kappa\left(x^{2}-1\right)
$$

and

$$
h_{y_{-}^{3}, y_{+}^{3}}\left(x^{1}, x^{2}+1\right)=k\left(x^{2}+1\right) \leq \kappa\left(x^{2}+1\right)
$$

therefore,

$$
h_{x_{-}^{3}, x_{+}^{3}}\left(x^{1}, x^{2}-1\right) \leq \kappa\left(x^{2}-1\right) \quad \text { and } \quad h_{y_{-}^{3}, y_{+}^{3}}\left(x^{1}, x^{2}-1\right) \geq \kappa\left(x^{2}-1\right) .
$$

By the intermediate value theorem of calculus, there is $\left(x_{-}, x_{+}\right) \in\left[x_{-}^{3}, y_{-}^{3}\right] \times$ $\left[x_{+}^{3}, y_{+}^{3}\right]$ such that $h_{x_{-}, x_{+}}\left(x^{1}, x^{2}-1\right)=\kappa\left(x^{2}-1\right)$, or equivalently [since $\left.h_{x_{-}, x_{+}}\left(x^{1}, x^{2}\right)=\kappa\left(x^{2}\right)\right]$, such that $h_{x_{-}, x_{+}}\left(x^{1}, \cdot\right)=\kappa(\cdot)$. Now let $h$ be the bilinear function equal to $h_{x_{-}, x_{+}}+c$ on $D$, where $c$ is the constant value of $g\left(x^{1}, \cdot\right)-\kappa(\cdot)$. Then $h\left(x^{1}, \cdot\right)=g\left(x^{1}, \cdot\right)$ and $h\left(x^{1} \pm 1, \cdot\right) \geq h_{x_{-}, x_{+}}\left(x^{1} \pm 1, \cdot\right) \geq g\left(x^{1} \pm 1, \cdot\right)$. Since $g$ is $h$-concave, $h \geq g$ on $D$ and $h$ is, therefore, a bilinear majorant of $f$ with a type 1 contact with $f$.

In the case where $x^{2}=N^{2}$, we let $x_{ \pm}^{3}$ be defined as above, but we set $y_{ \pm}^{3}=\infty$. Then (23) remains valid, as do the arguments that follow. The case where $x^{2}=0$ can be handled in a similar way.

We now prove that the condition concerning the solution of type 2 is necessary. Suppose that the solution is of type 2, and without loss of generality we assume that the hypotheses and conclusions of Proposition 3.4 are satisfied. Let $p^{1}, q^{1}, p^{2}$ and $q^{2}$ be defined as in (10) and (11), and let $g$ be the biconcave envelope of $f$. Then $N^{1}>p^{1}>q^{1}>0$ and after possibly transforming the problem by applying $\Phi_{1}$, we can assume, in addition, that $0<p^{2}<q^{2}<N^{2}$. Let $h$ be the unique bilinear function which coincides with $g$ on the four vertices of the rectangle $\left[q^{1}, p^{1}\right] \times\left[p^{2}, q^{2}\right]$. By Theorem 3.5 and 
Remark 3.2(b), $h$ and $g$ coincide in this rectangle. Since $g$ is $v$-concave, $h \geq g$ in $\left[q^{1}, p^{1}\right] \times\left[0, p^{2}\right]$. However, since $h=g$ on the segment $\left\{p^{1}\right\} \times\left[0, q^{2}\right]$ by Theorem 3.5, the $h$-concavity of $g$ implies that $h \geq g$ in $\left[0, p^{1}\right] \times\left[0, p^{2}\right]$. Notice also that $h\left(p^{1}, 0\right)=g\left(p^{1}, 0\right)=f\left(p^{1}, 0\right)$. By a similar argument, one shows that $h \geq g$ on each of the rectangles $\left[p^{1}, N^{1}\right] \times\left[0, q^{2}\right],\left[q^{1}, N^{1}\right] \times\left[q^{2}, N^{2}\right]$ and $\left[0, q^{1}\right] \times\left[p^{2}, N^{2}\right]$, which implies that $h \geq g$ in $D$ and, therefore, $h \geq f$ on $\partial D$. Since $h$ and $f$ agree at the four points $\left(p^{1}, 0\right),\left(N^{1}, q^{2}\right),\left(q^{1}, N^{2}\right)$ and $\left(0, p^{2}\right)$, $h$ is a bilinear majorant of $f$ and $\mathscr{C}_{1} \neq \varnothing, \ldots, b_{4} \neq \varnothing$, where $b_{1}, \ldots, b_{4}$ are the contact sets of $h$ with $f$. Since $p^{1}>q^{1}$, hypothesis (a) of Proposition 3.4 cannot hold. Together with (22), this implies that

$$
\mathscr{b}_{1} \cap \mathscr{b}_{3}=\varnothing \text {. }
$$

Since $p^{1} \in \mathscr{C}_{1}, q^{1} \in \mathscr{C}_{3}$ and both of these sets are intervals, we conclude from (24) that $\sup \mathscr{b}_{3}<\inf \mathscr{b}_{1}$. Since assumption (b) of Proposition 3.4 must hold, we conclude from an argument similar to the one used to prove (24) that $\mathscr{b}_{2} \cap \mathscr{C}_{4}=\varnothing$. Since $p^{2} \in \mathscr{C}_{4}$ and $q^{2} \in \mathscr{b}_{2}$, we conclude that inf $\mathscr{C}_{2}>\sup \mathscr{C}_{4}$. Therefore, $h$ has a type 2 contact with $f$.

We now prove that the condition concerning the solution of type 2 is sufficient. Assume that there exists a bilinear majorant $h$ of $f$ with a type 2 contact with $f$, and let $\mathscr{C}_{1}, \ldots, \mathscr{C}_{4}$ be the contact sets of $h$ with $f$. Set $x^{1}=\inf \mathscr{C}_{1}$, $y^{1}=\sup \mathscr{C}_{3}, x^{2}=\sup \mathscr{C}_{4}$ and $y^{2}=\inf \mathscr{C}_{2}$. Notice that $\left.x^{1}, y^{1} \in\right] 0, N^{1}[$ and $\left.x^{2}, y^{2} \in\right] 0, N^{2}[$. Without loss of generality, we can assume that

$$
y^{1}<x^{1} \text { and } x^{2}<y^{2} .
$$

Note that the inequality $h>f$ holds at the two points $\left(x^{1}-1,0\right)$ and $\left(y^{1}+\right.$ $1, N^{2}$ ).

In order to establish that the solution is of type 2, we first show that

$$
\left(\left\{x^{1}\right\} \times\right] 0, y^{2}[) \cup\left(\left\{y^{1}\right\} \times\right] x^{2}, N^{2}[) \subset \mathscr{E}_{2} .
$$

Let $S$ denote the set on the left-hand side and set $A_{1}=\left(x^{1}, x^{2}\right), A_{2}=\left(x^{1}, y^{2}\right)$, $A_{3}=\left(y^{1}, y^{2}\right), A_{4}=\left(y^{1}, x^{2}\right), A_{5}=A_{1}$ and $\xi_{1}=\left(x^{1}, 0\right), \xi_{2}=\left(N^{1}, y^{2}\right), \xi_{3}=$ $\left(y^{1}, N^{2}\right), \xi_{4}=\left(0, x^{2}\right)$. Since $g$ is a biconcave majorant of $f$, the four numbers $\lambda_{i}=g\left(A_{i}\right)$ satisfy the system of inequalities

$$
\lambda_{i} \geq \sigma_{i} f\left(\xi_{i}\right)+\rho_{i} \lambda_{i+1}, \quad i=1, \ldots, 4,
$$

where $\sigma_{i}=d\left(A_{i}, A_{i+1}\right) / d\left(A_{i+1}, \xi_{i}\right), \rho_{i}=1-\sigma_{i}$ and $d(\cdot, \cdot)$ denotes Euclidean distance. On the other hand, it is easy to check that the determinant of the system of equations

$$
\alpha_{i}=\sigma_{i} f\left(\xi_{i}\right)+\rho_{i} \alpha_{i+1}, \quad i=1, \ldots, 4,
$$

where $\alpha_{1}, \ldots, \alpha_{4}$ are the unknowns and $\alpha_{5}=\alpha_{1}$ by convention, is equal to $1-\rho_{1} \rho_{2} \rho_{3} \rho_{4}$. Since $\rho_{1} \rho_{2} \rho_{3} \rho_{4}<1$ by (25), this system has a unique solution, which is $\alpha_{i}=h\left(A_{i}\right)$ since $h$ is bilinear. Subtracting (28) from (27), we see that, for $i=1, \ldots, 4$,

$$
\lambda_{i}-\alpha_{i} \geq \rho_{i}\left(\lambda_{i+1}-\alpha_{i+1}\right) \geq \cdots \geq \rho_{1} \rho_{2} \rho_{3} \rho_{4}\left(\lambda_{i}-\alpha_{i}\right)
$$


and it follows that $\lambda_{i} \geq \alpha_{i}$. Therefore, $g\left(A_{i}\right) \geq h\left(A_{i}\right)$. Since $h$ is a biconcave majorant of $f, g \leq h$ and so $g\left(A_{i}\right)=h\left(A_{i}\right)$. Since $g\left(\xi_{i}\right)=h\left(\xi_{i}\right), i=1, \ldots, 4$, and $\mathrm{g}$ is $v$-concave, this implies that $g=h$ on $S$ and, therefore, $S \subset \mathscr{E}_{2}$. For future reference, we point out that we have also shown that

$$
(] 0, x^{1}\left[\times\left\{x^{2}\right\}\right) \cup(] y^{1}, N^{1}\left[\times\left\{y^{2}\right\}\right) \subset \mathscr{E}_{1}
$$

and, by Proposition 3.3, that

$$
g=h \quad \text { on }\left[y^{1}, x^{1}\right] \times\left[x^{2}, y^{2}\right] .
$$

We now show that hypothesis (b) of Proposition 3.4 is satisfied. If it were not, then there would exist $n \in] 0, N^{2}[$ such that $] 0, N^{1}\left[\times\{n\} \subset \mathscr{E}_{1}\right.$; that is,

$$
g(m, n)=\frac{N^{1}-m}{N^{1}} f(0, n)+\frac{m}{N^{1}} f\left(N^{1}, n\right) \quad \text { for all } m \in\left[0, N^{1}\right] .
$$

Suppose $\left.n \in] 0, x^{2}\right]$. Then $f(0, n) \leq h(0, n)$ since $h$ is a majorant of $f$, and $f\left(N^{1}, n\right)<h\left(N^{1}, n\right)$ since $n \leq x^{2}<y^{2}=\inf \mathscr{C}_{2}$. Since $\left(x^{1}, n\right) \in S$,

$$
g\left(x^{1}, n\right)<\frac{N^{1}-x^{1}}{N^{1}} h(0, n)+\frac{x^{1}}{N^{1}} h\left(N^{1}, n\right)=h\left(x^{1}, n\right)=g\left(x^{1}, n\right),
$$

a contradiction. The cases $n \in] x^{2}, y^{2}\left[\right.$ and $n \in\left[y^{2}, N^{2}[\right.$ lead in a similar way to a contradiction.

Consider now the functions $\mu_{1}$ and $\mu_{2}$ defined in (9) and the integers $p^{1}$, $q^{1}, p^{2}$ and $q^{2}$ defined in (10) and (11). Since $S \subset \mathscr{E}_{2}$, we conclude that $\mu_{1} \leq x^{1}$ and $\mu_{2} \geq y^{1}$. We are going to show that

$$
\sup \mu_{1}=x^{1} \text { and } \inf \mu_{2}=y^{1},
$$

which will complete the proof that the solution is of type 2. By hypothesis, $h\left(x^{1}-1,0\right)>f\left(x^{1}-1,0\right)=g\left(x^{1}-1,0\right)$, while $h\left(x^{1}-1, x^{2}\right)=g\left(x^{1}-1, x^{2}\right)$ by (30). There exists, therefore, $\left.n \in] 0, x^{2}\right]$ such that $g$ is strictly $v$-concave at $\left(x^{1}-1, n\right)$; in other words, $\left(x^{1}-1, n\right) \notin \mathscr{E}_{2}$. Since $\mathscr{E}_{2}$ is $h$-convex by Proposition 3.3 and since $\left(x^{1}, n\right) \in \mathscr{E}_{2}$, it follows that $(m, n) \notin \mathscr{E}_{2}$ for $0<m<x^{1}$. Consequently, $\mu_{1}(n)=x^{1}$ and, therefore, sup $\mu_{1}=x^{1}$. Similarly, one shows that inf $\mu_{2}=y^{1}$, and (31) is proved.

4.3. Determining the type of the solution. In this section, we give analytic conditions on the payoff function $f$ which ensure the existence of a bilinear majorant of $f$ with a contact of type 1 or a contact of type 2 with $f$. By Theorem 4.4, these conditions will tell us whether the solution is of type 1 or 2 . We shall also show how to construct this bilinear majorant and integers $p^{1}$, $q^{1}, p^{2}$ and $q^{2}$ that satisfy (10) and (11).

We let

$$
P_{1}=(0,0), \quad P_{2}=\left(N^{1}, 0\right), \quad P_{3}=\left(N^{1}, N^{2}\right), \quad P_{4}=\left(0, N^{2}\right)
$$

denote the four vertices of $D$. By convention, $P_{5}=P_{1}$ and $P_{0}=P_{4}$. 
Define eight functions $\phi_{i}^{j}, i=1, \ldots, 4, j=1,2$, as follows. First, the functions $\phi_{1}^{j}$ are the functions previously denoted $\phi^{j}, j=1,2$ :

$$
\begin{array}{ll}
\phi_{1}^{1}\left(x^{1}\right)=\left(x^{1}+1\right) f\left(x^{1}, 0\right)-x^{1} f\left(x^{1}+1,0\right) & \text { if } x^{1} \in\left[0, N^{1}[\right. \\
\phi_{1}^{2}\left(x^{2}\right)=\left(x^{2}+1\right) f\left(0, x^{2}\right)-x^{2} f\left(0, x^{2}+1\right) & \text { if } x^{2} \in\left[0, N^{2}[.\right.
\end{array}
$$

Next, in order to define $\phi_{i}^{j}, i=2,3,4, j=1,2$, we first apply to $D$ the transformation $\Phi_{1}, \Phi_{1} \circ \Phi_{2}$ or $\Phi_{1} \circ \Phi_{2} \circ \Phi_{1}$ to bring $P_{i}$ to the origin and then apply the formulas (32) to the transformed problem. For instance,

$$
\begin{array}{ll}
\phi_{4}^{2}\left(x^{2}\right)=\left(N^{2}-x^{2}+1\right) f\left(0, x^{2}\right)-\left(N^{2}-x^{2}\right) f\left(0, x^{2}-1\right) & \text { if } \left.\left.x^{2} \in\right] 0, N^{2}\right], \\
\phi_{3}^{1}\left(x^{1}\right)=\left(N^{1}-x^{1}+1\right) f\left(x^{1}, N^{2}\right)-\left(N^{1}-x^{1}\right) f\left(x^{1}-1, N^{2}\right) & \text { if } \left.\left.x^{1} \in\right] 0, N^{1}\right] .
\end{array}
$$

Notice that the subscript $i$ in the symbol $\phi_{i}^{j}$ refers to the vertex $P_{i}$ and the superscript $j$ to the horizontal direction $(j=1)$ or vertical direction $(j=2)$. Also, the geometric interpretation already mentioned in Remark 4.1 of the functions $\phi_{i}^{j}$ is important. For example, $\phi_{3}^{1}\left(x^{1}\right)$ is the value at $N^{1}$ of the unique linear function defined on $\left[0, N^{1}\right]$ whose value at $x^{1}$ is $f\left(x^{1}, N^{2}\right)$ and at $x^{1}-1$ is $f\left(x^{1}-1, N^{2}\right)$.

We now define four sets $\mathscr{D}_{1}, \ldots, \mathscr{D}_{4}$ as follows. The set $\mathscr{D}_{1}$ (resp. $\mathscr{D}_{3}$ ) is the set of all $x^{1} \in\left[0, N^{1}\left[\right.\right.$ (resp. $\left.\left.x^{1} \in\right] 0, N^{1}\right]$ ) such that the linear function defined on $\left[0, N^{2}\right]$ with value $\phi_{1}^{1}\left(x^{1}\right)$ at 0 and value $\phi_{4}^{1}\left(x^{1}\right)$ at $N^{2}$ [resp. value $\phi_{2}^{1}\left(x^{1}\right)$ at 0 and value $\phi_{3}^{1}\left(x^{1}\right)$ at $N^{2}$ ] is larger than $f(0, \cdot)$ [resp. $f\left(N^{1}, \cdot\right)$ ]. The set $\mathscr{D}_{2}$ (resp. $\left.\mathscr{D}_{4}\right)$ is the set of all $x^{2} \in\left[0, N^{2}\left[\left(\operatorname{resp} . x^{2} \in\right] 0, N^{2}\right]\right.$ ) such that the linear function defined on $\left[0, N^{1}\right]$ with value $\phi_{1}^{2}\left(x^{2}\right)$ at 0 and value $\phi_{2}^{2}\left(x^{2}\right)$ at $N^{1}$ [resp. value $\phi_{4}^{2}\left(x^{2}\right)$ at 0 and value $\phi_{3}^{2}\left(x^{2}\right)$ at $N^{1}$ ] is larger than $f(\cdot, 0)$ [resp. $\left.f\left(\cdot, N^{2}\right)\right]$.

Notice that if $x^{1} \in \mathscr{D}_{1}$, then $y^{1} \in \mathscr{D}_{1}$ whenever $y^{1}>x^{1}$. An analogous property holds for $\mathscr{D}_{i}, i=2,3,4$. We use the convention inf $\mathscr{D}_{1}=N^{1}$ if $\mathscr{D}_{1}=$ $\varnothing$, inf $\mathscr{D}_{2}=N^{2}$ if $\mathscr{D}_{2}=\varnothing, \sup \mathscr{D}_{3}=0$ if $\mathscr{D}_{3}=\varnothing$ and $\sup \mathscr{D}_{4}=0$ if $\mathscr{D}_{4}=\varnothing$. Given the payoff function $f$, the infima and suprema of $\mathscr{D}_{1}, \ldots, \mathscr{D}_{4}$ are easy to determine.

REMARK 4.5. Let $x^{1}=\inf \mathscr{D}_{1}$ and suppose $x^{1} \geq 1$. Then the set $\left\{x^{2} \in\right.$ ] $0, N^{2}\left[: \phi_{1}^{2}\left(x^{2}\right)>\phi_{1}^{1}\left(x^{1}-1\right)\right\}$ is not empty and the infimum $y^{2}$ of this set satisfies

$$
\phi_{4}^{2}\left(y^{2}\right)>\phi_{4}^{1}\left(x^{1}-1\right)
$$

Indeed, the set in question contains $N^{2}-1$, since otherwise $\phi_{1}^{2}\left(N^{2}-1\right) \leq$ $\phi_{1}^{1}\left(x^{1}-1\right)$ and, therefore, the linear function $h$ defined on $\left[0, N_{2}\right]$ with value $\phi_{1}^{1}\left(x^{1}-1\right)$ at 0 and value $\phi_{4}^{1}\left(x^{1}-1\right)$ at $N^{2}$ would dominate $f(0, \cdot)$, contradicting the fact that $x^{1}-1 \notin \mathscr{D}_{1}$. Now if $\phi_{4}^{2}\left(y^{2}\right) \leq \phi_{4}^{1}\left(x^{1}-1\right)$, then since $\phi_{1}^{2}\left(y^{2}-1\right) \leq$ $\phi_{1}^{1}\left(x^{1}-1\right)$, the function $h$ would dominate $f(0, \cdot)$, a contradiction. 
Given the four sets $\mathscr{D}_{1}, \ldots, \mathscr{D}_{4}$, we consider two cases.

CASE A. $\quad \inf \mathscr{D}_{1}<\sup \mathscr{D}_{3}$ or $\inf \mathscr{D}_{2}<\sup \mathscr{D}_{4}$.

CASE B. $\inf \mathscr{D}_{1}>\sup \mathscr{D}_{3}$ and $\inf \mathscr{D}_{2}>\sup \mathscr{D}_{4}$.

In either Case $\mathrm{A}$ or $\mathrm{B}$, we shall show how to determine the type of the solution. If neither Case A nor Case B holds, then inf $\mathscr{D}_{1} \geq \sup \mathscr{D}_{3}$, inf $\mathscr{D}_{2} \geq$ sup $\mathscr{D}_{4}$ and one or both of these two inequalities is an equality. This is a critical case, in which determining the type of the solution requires additional work.

The next theorem shows how to solve the optimal switching problem when Case A holds.

THEOREM 4.6. If Case A holds, then the solution to the optimal switching problem is of type 1. In this case, after possibly transforming the problem by applying $\Phi_{2}$, the inequality inf $\mathscr{D}_{1}<\sup \mathscr{D}_{3}$ holds and hypothesis (a) of Proposition 3.4 is satisfied. In addition, the integers $p^{1}, q^{1}, p^{2}$ and $q^{2}$ defined by

$$
\begin{aligned}
& \left.\begin{array}{l}
p^{1}=\inf \mathscr{D}_{1} \\
p^{2}=\inf \left\{x^{2} \in\right] 0, N^{2}\left[: \phi_{1}^{2}\left(x^{2}\right)>\phi_{1}^{1}\left(p^{1}-1\right)\right\}
\end{array}\right\} \quad \text { if } \inf \mathscr{D}_{1}>0, \\
& \left.\begin{array}{l}
p^{1}=1 \\
p^{2}=1
\end{array}\right\} \quad \text { if } \inf \mathscr{D}_{1}=0 \text {, } \\
& \left.\begin{array}{l}
q^{1}=\sup \mathscr{D}_{3} \\
q^{2}=\sup \left\{x^{2} \in\right] 0, N^{2}\left[: \phi_{3}^{2}\left(x^{2}\right)>\phi_{3}^{1}\left(q^{1}+1\right)\right\}
\end{array}\right\} \quad \text { if } \sup \mathscr{D}_{3}<N^{1}, \\
& \left.\begin{array}{l}
q^{1}=N^{1}-1 \\
q^{2}=1
\end{array}\right\} \quad \text { if } \sup \mathscr{D}_{3}=N^{1},
\end{aligned}
$$

satisfy (10) and (11).

Proof. If inf $\mathscr{D}_{2}<\sup \mathscr{D}_{4}$, then inf $\mathscr{D}_{1}<\sup \mathscr{D}_{3}$ for the transformation of the problem under $\Phi_{2}$. So we let $\tilde{p}^{1}=\inf \mathscr{D}_{1}, \tilde{q}^{1}=\sup \mathscr{D}_{3}$ and assume that $\tilde{p}^{1}<\tilde{q}^{1}$. For $x^{1} \in\left[\tilde{p}^{1}, \tilde{q}^{1}[\right.$, let $h$ be the unique bilinear function on $D$ that agrees with $f$ at the four vertices of the rectangle $\left[x^{1}, x^{1}+1\right] \times\left[0, N^{2}\right]$. Since $x^{1} \in \mathscr{D}_{1}, h$ dominates $f$ on $\left[0, x^{1}\right] \times\left[0, N^{2}\right]$. Since $x^{1}+1 \leq \tilde{q}^{1}$ and $\tilde{q}^{1} \in \mathscr{D}_{3}$, we conclude that $x^{1}+1$ belongs to $\mathscr{D}_{3}$, which means that $h$ also dominates $f$ on $\left[x^{1}+1, N^{1}\right] \times\left[0, N^{2}\right]$. Since either $x^{1}$ or $x^{1}+1$ belongs to $] 0, N^{1}[, h$ is a bilinear majorant of $f$ with a type 1 contact with $f$, so the solution is of type 1 by Theorem 4.4 .

These considerations and (22) also show that the biconcave envelope $g$ of $f$ is $v$-linear on $\left[\tilde{p}^{1}, \tilde{q}^{1}\right] \times\left[0, N^{2}\right]$. In particular, hypothesis (a) of Proposition 3.4 is satisfied and the integer $p^{1}$ defined in (10) satisfies $1 \leq p^{1} \leq \tilde{p}^{1}$ if $\tilde{p}^{1} \geq 1$ and $p^{1}=1$ if $\tilde{p}^{1}=0$. When $p^{1}=1, p^{2}=1$ satisfies (11). In order to prove 
that $p^{1}=\tilde{p}^{1}$ when $\tilde{p}^{1} \geq 1$, we only need to show that if $\tilde{p}^{1}>1$, then there is $\left.x^{2} \in\right] 0, N^{2}\left[\right.$ such that $\left(\tilde{p}^{1}-1, x^{2}\right) \in \mathscr{E}_{1} \backslash \mathscr{E}_{2}$. Let $h$ be the bilinear function which agrees with $f$ at the four vertices of the rectangle $R=\left[\tilde{p}^{1}-1, \tilde{p}^{1}\right] \times\left[0, N^{2}\right]$. Notice that $h$ does not dominate $g$ on $R$. Indeed, otherwise, $h$ would agree with $g$ on this rectangle, and since $g$ is $h$-concave, $h$ would dominate $g$ in $D$; therefore, $h(0, \cdot)$ would dominate $f(0, \cdot)$. Since $h(0,0)=\phi_{1}^{1}\left(\tilde{p}^{1}-1\right)$ and $h\left(0, N^{2}\right)=\phi_{4}^{1}\left(\tilde{p}^{1}-1\right)$, this would contradict the fact that $\tilde{p}^{1}-1$ does not belong to $\mathscr{D}_{1}$. It follows that $h<g$ at some point of the segment $\left.\left\{\tilde{p}^{1}-1\right\} \times\right] 0, N^{2}[$, so $g\left(\tilde{p}^{1}-1, \cdot\right)$ is not linear. This means that there is $\left.x^{2} \in\right] 0, N^{2}[$ such that $\left(\tilde{p}^{1}-1, x^{2}\right) \notin \mathscr{E}_{2}$. Since $\mathscr{E}_{1} \cup \mathscr{E}_{2}=D^{\circ}$, this element belongs to $\mathscr{E}_{1} \backslash \mathscr{E}_{2}$.

To complete the proof of (33), assume inf $\mathscr{D}_{1}>0$ and let $\tilde{p}^{2}$ denote the right-hand side of the second equality in (33). Notice that the set over which the infimum is taken is not empty, by Remark 4.5. All we now need to show is that $\mu_{1}\left(\tilde{p}^{2}\right)=p^{1}$, where $\mu_{1}$ is the function defined in (9). Let $p^{2}$ be as in (11). If $\tilde{p}^{2}=p^{2}$, then this is true by the choice of $p^{2}$. If $\tilde{p}^{2}<p^{2}$, then since $\phi_{1}^{2}\left(\tilde{p}^{2}\right)>\phi_{1}^{1}\left(p^{1}-1\right)$ and $\phi_{1}^{2}\left(\tilde{p}^{2}-1\right) \leq \phi_{1}^{1}\left(p^{1}-1\right)$, we see that $\xi_{1}\left(\tilde{p}^{2}\right)=p^{1}$ and $\xi_{1}\left(\tilde{p}^{2}-1\right) \leq p^{1}-1$ by Theorem 4.2 . By $(12)$, it follows that $\mu_{1}\left(\tilde{p}^{2}\right)=p^{1}$. To complete the proof, it suffices to establish that the inequality $p^{2} \geq \tilde{p}^{2}$ holds. Since $p^{1}=\inf \mathscr{D}_{1} \geq 1$, we conclude from Remark 4.5 that

$$
\phi_{4}^{2}\left(\tilde{p}^{2}\right)>\phi_{4}^{1}\left(p^{1}-1\right) .
$$

Assume now that $p^{2}<\tilde{p}^{2}$. Applying the statement analogous to Theorem 4.2 for the interval $] p^{2}, N^{2}\left[\right.$, we see from (35) that $\xi_{1}\left(\tilde{p}^{2}\right)=p^{1}$; therefore, $] 0, p^{1}\left[\times\left\{\tilde{p}^{2}\right\} \subset \mathscr{E}_{1}\right.$. Since $] 0, p^{1}\left[\times\left\{p^{2}\right\} \subset \mathscr{E}_{1}\right.$ and $\mathscr{E}_{1}$ is $v$-convex by Proposition 3.3,$] 0, p^{1}\left[\times\left[p^{2}, \tilde{p}^{2}\right] \subset \mathscr{E}_{1}\right.$. Therefore, $g$ would be $h$-linear on $\left[0, p^{1}\right] \times\left[p^{2}, \tilde{p}^{2}\right]$. In particular, the bilinear function $h$ which agrees with $g$ on $\left[0, p^{1}\right] \times\left[\tilde{p}^{2}-1, \tilde{p}^{2}\right]$ would dominate $g$ on $\left[0, p^{1}\right] \times\left[0, \tilde{p}^{2}\right]$ since $g$ is $v$-concave. Since $g$ is strictly $v$-concave at $\left(p^{1}-1, p^{2}\right)$ by $(11)$, the inequality $f\left(p^{1}-1,0\right)<h\left(p^{1}-1,0\right)$ would hold, or equivalently, $h(0,0)>\phi_{1}^{1}\left(p^{1}-1\right)$ [since $\left.f\left(p^{1}, 0\right)=h\left(p^{1}, 0\right)\right]$. Since $h(0,0)$ is also equal to $\phi_{1}^{2}\left(\tilde{p}^{2}-1\right) \leq \phi_{1}^{1}\left(p^{1}-1\right)$ by definition of $\tilde{p}^{2}$, we have derived a contradiction from the assumption.

The proof of (34) is similar and is left to the reader.

To handle Case $\mathrm{B}$, we now define for $i=1, \ldots, 4$ a transformation $T_{i}:\left[f\left(P_{i}\right), \infty\left[\rightarrow \mathbb{R}\right.\right.$. We give the definition for $T_{1}$, since $T_{2}, T_{3}$ and $T_{4}$ are defined analogously. For $x^{3} \in\left[f\left(P_{i}\right), \infty[\right.$, let $h$ denote the linear function defined on $\left[0, N^{1}\right]$ such that $h \geq f(\cdot, 0), h(0)=x^{3}$ and $h\left(x^{1}\right)=f\left(x^{1}, 0\right)$ for at least one $\left.x^{1} \in\right] 0, N^{1}$ ] [if $x^{3}=f\left(P_{1}\right)$, then $x^{1}$ may be taken equal to 1 ]. Then $T_{1}\left(x^{3}\right)$ is by definition equal to $h\left(N^{1}\right)$.

It is clear that $T_{i}$ is continuous, nonincreasing and even strictly decreasing on the interval $\left[f\left(P_{i}\right), m_{i}\right]$, where $m_{1}=\phi_{1}^{1}\left(N^{1}-1\right), m_{2}=\phi_{2}^{2}\left(N^{2}-1\right), m_{3}=$ $\phi_{3}^{1}(1)$ and $m_{4}=\phi_{4}^{2}(1)$. Also, observe that

$$
\begin{array}{ll}
T_{1}\left(\phi_{1}^{1}\left(x^{1}\right)\right)=\phi_{2}^{1}\left(x^{1}+1\right), & T_{2}\left(\phi_{2}^{2}\left(x^{2}\right)\right)=\phi_{3}^{2}\left(x^{2}+1\right), \\
T_{3}\left(\phi_{3}^{1}\left(x^{1}\right)\right)=\phi_{4}^{1}\left(x^{1}-1\right), & T_{4}\left(\phi_{4}^{2}\left(x^{2}\right)\right)=\phi_{1}^{2}\left(x^{2}-1\right) .
\end{array}
$$


Another property of the transformation $T_{1}$ is that

$$
0 \leq x^{2}<\inf \mathscr{D}_{2} \quad \text { implies } \phi_{1}^{2}\left(x^{2}\right)<T_{1}^{-1}\left(\phi_{2}^{2}\left(x^{2}\right)\right) .
$$

Indeed, if $\phi_{1}^{2}\left(x^{2}\right)$ were greater than or equal to $T_{1}^{-1}\left(\phi_{2}^{2}\left(x^{2}\right)\right)$, then $T_{1}\left(\phi_{1}^{2}\left(x^{2}\right)\right) \leq$ $\phi_{2}^{2}\left(x^{2}\right)$, which would imply that $x^{2} \in \mathscr{D}_{2}$, a contradiction.

The transformation

$$
T=T_{4} \circ T_{3} \circ T_{2} \circ T_{1}
$$

will play an important role below. Indeed, if $x_{1}^{3}$ is a fixed point of $T$ and if $x_{i+1}^{3}=T_{i}\left(x_{i}^{3}\right), i=1,2,3$, then the unique bilinear function $h$ whose value at $P_{i}$ is $x_{i}^{3}, i=1, \ldots, 4$, is termed the bilinear function associated with this fixed point. It is a bilinear majorant of $f$ and if $x_{i}^{3}>f\left(P_{i}\right), i=1, \ldots, 4$, then all four contact sets of $h$ with $f$ are nonempty.

The following theorem shows how to solve the optimal switching problem when Case B holds.

THEOREM 4.7. In Case $\mathrm{B}$, the solution to the optimal switching problem is of type 2. Let $b_{1}^{1}=\inf \mathscr{D}_{1}$ and $b_{2}^{2}=\inf \mathscr{D}_{2}$. The transformation $T$ has a unique fixed point $x^{3}$ in the interval $I_{1}=\left[\phi_{1}^{1}\left(b_{1}^{1}-1\right), T_{1}^{-1}\left(\phi_{2}^{2}\left(b_{2}^{2}-1\right)\right)\right]$ and the bilinear function $h$ associated with $x^{3}$ is a bilinear majorant of $f$ with a type 2 contact with $f$. This fixed point is characterized by the property that, for $y_{1} \in I_{1}, x^{3}>T\left(y_{1}\right)>y_{1}$ if $y_{1}<x^{3}$, and $x^{3}<T\left(y_{1}\right)<y_{1}$ if $y_{1}>x^{3}$. If $\mathscr{C}_{1}, \ldots, \mathscr{C}_{4}$ are the contact sets of $h$ with $f$, then after possibly transforming the problem by applying $\Phi_{1}$ the inequality inf $\mathscr{C}_{1}>\sup \mathscr{C}_{3}$ holds and hypothesis (b) of Proposition 3.4 is satisfied. In addition, the integers $p^{1}, q^{1}, p^{2}$ and $q^{2}$ defined by

$$
p^{1}=\inf \mathscr{C}_{1}, \quad q^{1}=\sup \mathscr{C}_{3}, \quad p^{2}=\inf \mathscr{C}_{4}, \quad q^{2}=\sup \mathscr{C}_{2},
$$

satisfy (10) and (11).

Proof. Assume Case B holds and define four subsets of $D$ by setting

$$
\begin{aligned}
& D_{1}=\left\{\left(x^{1}, x^{2}\right): \phi_{4}^{2}\left(x^{2}\right)>\phi_{4}^{1}\left(x^{1}\right) \text { and } \phi_{1}^{2}\left(x^{2}\right)>\phi_{1}^{1}\left(x^{1}\right)\right\}, \\
& D_{2}=\left\{\left(x^{1}, x^{2}\right): \phi_{1}^{1}\left(x^{1}\right)>\phi_{1}^{2}\left(x^{2}\right) \text { and } \phi_{2}^{1}\left(x^{1}\right)>\phi_{2}^{2}\left(x^{2}\right)\right\}, \\
& D_{3}=\left\{\left(x^{1}, x^{2}\right): \phi_{2}^{2}\left(x^{2}\right)>\phi_{2}^{1}\left(x^{1}\right) \text { and } \phi_{3}^{2}\left(x^{2}\right)>\phi_{3}^{1}\left(x^{1}\right)\right\}, \\
& D_{4}=\left\{\left(x^{1}, x^{2}\right): \phi_{3}^{1}\left(x^{1}\right)>\phi_{3}^{2}\left(x^{2}\right) \text { and } \phi_{4}^{1}\left(x^{1}\right)>\phi_{4}^{2}\left(x^{2}\right)\right\} .
\end{aligned}
$$

Notice that

$$
D_{1} \cap D_{2}=\varnothing, \quad D_{2} \cap D_{3}=\varnothing, \quad D_{3} \cap D_{4}=\varnothing, \quad D_{4} \cap D_{1}=\varnothing .
$$

Since each function $\phi_{i}^{j}$ is monotone, it is easy to check that

$$
\text { each set } D_{i} \text { is enclosed by the graph of a unimodal function }
$$


(and by a segment of $\partial D$ ). Let $b_{3}^{1}=\sup \mathscr{D}_{3}$ and $b_{4}^{2}=\sup \mathscr{D}_{4}$. Given that $\inf \mathscr{D}_{1}>\sup \mathscr{D}_{3}$, then in particular, $b_{1}^{1} \geq 1$ and $b_{3}^{1} \leq N^{1}-1$ since $N^{1} \geq b_{1}^{1}>$ $b_{3}^{1} \geq 0$. Similarly, $b_{2}^{2} \geq 1$ and $b_{4}^{2} \leq N^{2}-1$. Set

$$
\begin{aligned}
& b_{1}^{2}=\inf \left\{x^{2} \in\right] 0, N^{2}\left[: \phi_{1}^{2}\left(x^{2}\right)>\phi_{1}^{1}\left(b_{1}^{1}-1\right)\right\}, \\
& b_{2}^{1}=\sup \left\{x^{1} \in\right] 0, N^{1}\left[: \phi_{2}^{1}\left(x^{1}\right)>\phi_{2}^{2}\left(b_{2}^{2}-1\right)\right\}, \\
& b_{3}^{2}=\sup \left\{x^{2} \in\right] 0, N^{2}\left[: \phi_{3}^{2}\left(x^{2}\right)>\phi_{3}^{1}\left(b_{3}^{1}+1\right)\right\}, \\
& b_{4}^{1}=\inf \left\{x^{1} \in\right] 0, N^{1}\left[: \phi_{4}^{1}\left(x^{1}\right)>\phi_{4}^{2}\left(b_{4}^{2}+1\right)\right\}
\end{aligned}
$$

For reasons similar to the one which showed that the set appearing in Remark 4.5 is not empty, the sets on each right-hand side above are nonempty. Notice that

$$
\begin{array}{ll}
\left(b_{1}^{1}-1, b_{1}^{2}\right) \in D_{1}, & \left(b_{2}^{1}, b_{2}^{2}-1\right) \in D_{2}, \\
\left(b_{3}^{1}+1, b_{3}^{2}\right) \in D_{3}, & \left(b_{4}^{1}, b_{4}^{2}+1\right) \in D_{4} .
\end{array}
$$

Indeed, the first relation is true since $\phi_{1}^{2}\left(b_{1}^{2}\right)>\phi_{1}^{1}\left(b_{1}^{1}-1\right)$ by the definition of $b_{1}^{2}$ and $\phi_{4}^{2}\left(b_{1}^{2}\right)>\phi_{4}^{1}\left(b_{1}^{1}-1\right)$ by Remark 4.5 . The other three relations can be checked in a similar way.

Figure 2 shows a possible disposition of the sets $D_{1}, \ldots, D_{4}$. Since $b_{2}^{2}>b_{4}^{2}$, $D_{1}$ cannot intersect $D_{3}$ by (39) and (40). Similarly, $D_{2}$ cannot intersect $D_{4}$, and so $D_{1}, \ldots, D_{4}$ are disjoint. From the figure, notice that we can assume that $b_{1}^{2} \leq b_{3}^{2}$, since otherwise this would be the case after applying $\Phi_{1}$. Also from the figure and (40), notice that the inequalities $b_{1}^{1}>b_{3}^{1}$ and $b_{4}^{2}<b_{2}^{2}$ imply that $b_{4}^{1} \leq b_{3}^{1}, b_{1}^{1} \leq b_{2}^{1}, b_{1}^{2} \leq b_{4}^{2}$ and $b_{2}^{2} \leq b_{3}^{2}$ and, therefore,

$$
0<b_{4}^{1} \leq b_{3}^{1}<b_{1}^{1} \leq b_{2}^{1}<N^{1} \text { and } 0<b_{1}^{2} \leq b_{4}^{2}<b_{2}^{2} \leq b_{3}^{2}<N^{2} .
$$

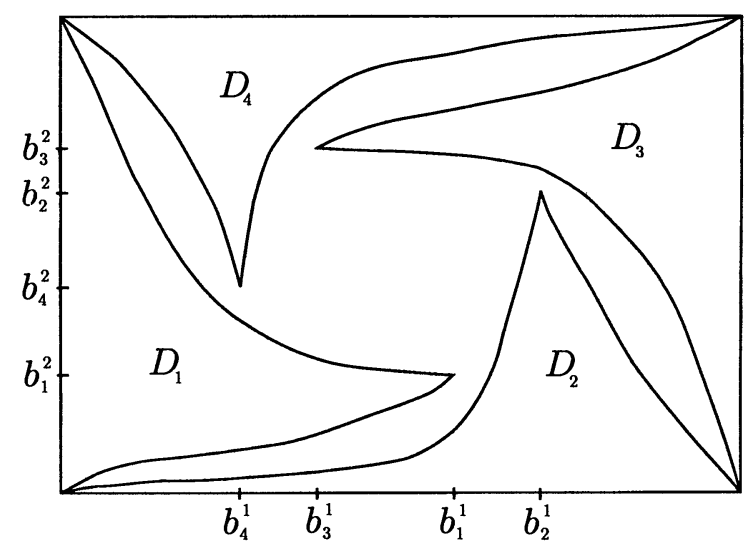

FIG. 2. The sets $D_{1}, \ldots, D_{4}$. 
Since $b_{1}^{1} \leq b_{2}^{1}$ and $\phi_{2}^{1}\left(b_{2}^{1}\right)>\phi_{2}^{2}\left(b_{2}^{2}-1\right)$, it follows from (36) that

$$
T_{1}\left(\phi_{1}^{1}\left(b_{1}^{1}-1\right)\right)=\phi_{2}^{1}\left(b_{1}^{1}\right) \geq \phi_{2}^{1}\left(b_{2}^{1}\right)>\phi_{2}^{2}\left(b_{2}^{2}-1\right)
$$

and, therefore, that

$$
\phi_{1}^{1}\left(b_{1}^{1}-1\right)<T_{1}^{-1}\left(\phi_{2}^{2}\left(b_{2}^{2}-1\right)\right),
$$

and one checks in a similar way that

$$
\begin{aligned}
& \phi_{2}^{2}\left(b_{2}^{2}-1\right)<T_{2}^{-1}\left(\phi_{3}^{1}\left(b_{3}^{1}+1\right)\right), \\
& \phi_{3}^{1}\left(b_{3}^{1}+1\right)<T_{3}^{-1}\left(\phi_{4}^{2}\left(b_{4}^{2}+1\right)\right), \\
& \phi_{4}^{2}\left(b_{4}^{2}+1\right)<T_{4}^{-1}\left(\phi_{1}^{1}\left(b_{1}^{1}-1\right)\right) .
\end{aligned}
$$

Define four intervals of $\mathbb{R}$ by setting

$$
\begin{array}{ll}
I_{1}=\left[\phi_{1}^{1}\left(b_{1}^{1}-1\right), T_{1}^{-1}\left(\phi_{2}^{2}\left(b_{2}^{2}-1\right)\right)\right], & I_{2}=\left[\phi_{2}^{2}\left(b_{2}^{2}-1\right), T_{2}^{-1}\left(\phi_{3}^{1}\left(b_{3}^{1}+1\right)\right)\right], \\
I_{3}=\left[\phi_{3}^{1}\left(b_{3}^{1}+1\right), T_{3}^{-1}\left(\phi_{4}^{2}\left(b_{4}^{2}+1\right)\right)\right], & I_{4}=\left[\phi_{4}^{2}\left(b_{4}^{2}+1\right), T_{4}^{-1}\left(\phi_{1}^{1}\left(b_{1}^{1}-1\right)\right)\right] .
\end{array}
$$

Moreover, set $I_{5}=I_{1}$. Since $b_{3}^{1}<b_{1}^{1}$,

$$
T_{1}\left(\phi_{1}^{1}\left(b_{1}^{1}-1\right)\right) \leq T_{1}\left(\phi_{1}^{1}\left(b_{3}^{1}\right)\right)=\phi_{2}^{1}\left(b_{3}^{1}+1\right)<T_{2}^{-1}\left(\phi_{3}^{1}\left(b_{3}^{1}+1\right)\right),
$$

where the equality follows from (36) and the last inequality from the property of $T_{2}$ analogous to (37). Similarly,

$$
\begin{aligned}
& T_{2}\left(\phi_{2}^{2}\left(b_{2}^{2}-1\right)\right)<T_{3}^{-1}\left(\phi_{4}^{2}\left(b_{4}^{2}+1\right)\right), \\
& T_{3}\left(\phi_{3}^{1}\left(b_{3}^{1}+1\right)\right)<T_{4}^{-1}\left(\phi_{1}^{1}\left(b_{1}^{1}-1\right)\right), \\
& T_{4}\left(\phi_{4}^{2}\left(b_{4}^{2}+1\right)\right)<T_{1}^{-1}\left(\phi_{2}^{2}\left(b_{2}^{2}-1\right)\right) .
\end{aligned}
$$

Since $T_{i}$ is nonincreasing, we conclude that $T_{i}\left(I_{i}\right) \subset I_{i+1}, i=1, \ldots, 4$. Consequently, $T\left(I_{1}\right) \subset I_{1}$ and so $T$ admits a fixed point $x^{3}$ in $I_{1}$. Let $h$ be the bilinear function associated with $x^{3}$ and set $x_{1}^{3}=x^{3}, x_{i+1}^{3}=T_{i}\left(x_{i}^{3}\right), i=1,2,3$. Notice that $x_{1}^{3} \in T_{4}\left(I_{4}\right)$, so

$$
x_{1}^{3} \leq T_{4}\left(\phi_{4}^{2}\left(b_{4}^{2}+1\right)\right)<T_{1}^{-1}\left(\phi_{2}^{2}\left(b_{2}^{2}-1\right)\right)
$$

by (46) and, therefore,

$$
x_{2}^{3}=T_{1}\left(x_{1}^{3}\right)>\phi_{2}^{2}\left(b_{2}^{2}-1\right) .
$$

Similarly, one shows that

$$
x_{2}^{3}<T_{2}^{-1}\left(\phi_{3}^{1}\left(b_{3}^{1}+1\right)\right), \quad x_{3}^{3}<T_{3}^{-1}\left(\phi_{4}^{2}\left(b_{4}^{2}+1\right)\right), \quad x_{4}^{3}<T_{4}^{-1}\left(\phi_{1}^{1}\left(b_{1}^{1}-1\right)\right)
$$

and

$$
x_{3}^{3}>\phi_{3}^{1}\left(b_{3}^{1}+1\right), \quad x_{4}^{3}>\phi_{4}^{2}\left(b_{4}^{2}+1\right), \quad x_{1}^{3}>\phi_{1}^{1}\left(b_{1}^{1}-1\right) .
$$

Therefore, the contact sets $\mathscr{C}_{1}, \ldots, \mathscr{C}_{4}$ of $h$ with $f$ are nonempty and satisfy

$$
\inf \mathscr{C}_{1} \geq b_{1}^{1}, \quad \inf \mathscr{C}_{2} \geq b_{2}^{2}, \quad \sup \mathscr{C}_{3} \leq b_{3}^{1}, \quad \sup \mathscr{C}_{4} \leq b_{4}^{2} .
$$


By (43), this implies that $h$ has a type 2 contact with $f$, and so the solution is of type 2 by Theorem 4.2. Using the argument which follows (30), we see that assumption (b) of Proposition 3.4 is satisfied.

We shall now prove that $T$ has a unique fixed point in $I_{1}$. Fix $y_{1} \in I_{1}$. Consider the linear function defined on $\left[0, N^{1}\right]$ which is equal to $y_{1}$ at $P_{1}$ and to $T_{1}\left(y_{1}\right)$ at $N^{1}$, and suppose that the interval on which this linear function agrees with $f(\cdot, 0)$ is $\left[\alpha_{1}\left(y_{1}\right), \beta_{1}\left(y_{1}\right)\right]$ [this interval may only contain a single point, in which case $\left.\alpha_{1}\left(y_{1}\right)=\beta_{1}\left(y_{1}\right)\right]$. It is not difficult to deduce from the definition of $T_{1}$ that

$$
T_{1}\left(y_{1}\right)=y_{1}+N^{1} \frac{f\left(\beta_{1}\left(y_{1}\right), 0\right)-y_{1}}{\beta_{1}\left(y_{1}\right)} .
$$

Since $\beta_{1}(\cdot)$ is constant on the interval $\left[y_{1}, y_{1}+\varepsilon\right]$ for some $\varepsilon>0$, the right derivative $D_{+} T_{1}\left(y_{1}\right)$ is equal to

$$
D_{+} T_{1}\left(y_{1}\right)=1-\frac{N^{1}}{\beta_{1}\left(y_{1}\right)} .
$$

Similarly, the left derivative of $T_{1}$ at $y_{1}$ is $D_{-} T_{1}\left(y_{1}\right)=1-N^{1} / \alpha_{1}\left(y_{1}\right)$. If for $y_{i} \in I_{i}$ we define intervals $\left[\alpha_{i}\left(y_{i}\right), \beta_{i}\left(y_{i}\right)\right]$ relative to $T_{i}$ by analogy with the above, then similar formulas hold for $D_{ \pm} T_{i}\left(y_{i}\right), i=2,3,4$.

Since the $T_{i}$ are decreasing, the chain rule for derivatives, applied to $T$, yields

$$
D_{+} T\left(y_{1}\right)=D_{-} T_{4}\left(y_{4}\right) D_{+} T_{3}\left(y_{3}\right) D_{-} T_{2}\left(y_{2}\right) D_{+} T_{1}\left(y_{1}\right),
$$

and this is equal to

$$
\left(1-\frac{N^{2}}{N^{2}-\beta_{4}\left(y_{4}\right)}\right)\left(1-\frac{N^{1}}{N^{1}-\alpha_{3}\left(y_{3}\right)}\right)\left(1-\frac{N^{2}}{\alpha_{2}\left(y_{2}\right)}\right)\left(1-\frac{N^{1}}{\beta_{1}\left(y_{1}\right)}\right),
$$

which, after reducing each factor to a common denominator, equals

$$
\begin{gathered}
\frac{\alpha_{3}\left(y_{3}\right)}{\beta_{1}\left(y_{1}\right)} \frac{N^{1}-\beta_{1}\left(y_{1}\right)}{N^{1}-\alpha_{3}\left(y_{3}\right)} \frac{\beta_{4}\left(y_{4}\right)}{\alpha_{2}\left(y_{2}\right)} \frac{N^{2}-\alpha_{2}\left(y_{2}\right)}{N^{2}-\beta_{4}\left(y_{4}\right)} \\
\leq \frac{b_{3}^{1}}{b_{1}^{1}} \frac{N^{1}-b_{1}^{1}}{N^{1}-b_{3}^{1}} \frac{b_{4}^{2}}{b_{2}^{2}} \frac{N^{2}-b_{2}^{2}}{N^{2}-b_{4}^{2}}<1
\end{gathered}
$$

since $y_{1} \in I_{1}$ and since we are in Case B. Consequently, $D_{+} T<1$ in $I_{1}$ and so $T$ has at most one fixed point $x^{3}$ in $I_{1}$. Since $T$ is nondecreasing, this also shows that, for $y_{1} \in I_{1}, x^{3}>T\left(y_{1}\right)>y_{1}$ if $y_{1}<x^{3}$, and $x^{3}<T\left(y_{1}\right)<y_{1}$ if $y_{1}>x^{3}$. Clearly, this property can only be satisfied by the (unique) fixed point of $T$.

Set $p^{1}=\inf \mathscr{C}_{1}, q^{1}=\sup \mathscr{C}_{3}, p^{2}=\inf \mathscr{C}_{4}$ and $q^{2}=\sup \mathscr{C}_{2}$. If $p^{2}-1>0$ and $q^{2}+1<N^{2}$, then

$$
h\left(0, p^{2}-1\right)>f\left(0, p^{2}-1\right) \text { and } h\left(N^{1}, q^{2}+1\right)>f\left(N^{1}, q^{2}+1\right) .
$$


By (30), $g=h$ on $\left[q^{1}, p^{1}\right] \times\left[p^{2}, q^{2}\right]$, and by (26) and (29),

$$
\left(\left\{p^{1}\right\} \times\right] 0, q^{2}[) \cup\left(\left\{q^{1}\right\} \times\right] p^{2}, N^{2}[) \subset \mathscr{E}_{2}
$$

and

$$
(] 0, p^{1}\left[\times\left\{p^{2}\right\}\right) \cup(] q^{1}, N^{1}\left[\times\left\{q^{2}\right\}\right) \subset \mathscr{E}_{1} .
$$

Let $\mu_{1}$ and $\mu_{2}$ be the functions defined in (9). By (31), $p^{1}=\sup \mu_{1}$ and $q^{1}=$ inf $\mu_{2}$, so (10) is satisfied. By (50), $\mu_{1}(n) \leq q^{1}$ for $n>p^{2}$ and so there is $\left.n \in] 0, p^{2}\right]$ such that $\mu_{1}(n)=p^{1}$. If $n=p^{2}$, then $\mu_{1}\left(p^{2}\right)=p^{1}$; in other words, $p^{2}$ satisfies (11). Suppose $\left.n \in\right] 0, p^{2}$ [. To conclude that $\mu_{1}\left(p^{2}\right)=p^{1}$, we must show that $\left(p^{1}-1, p^{2}\right) \notin \mathscr{E}_{2}$. Indeed, since $\left(p^{1}, p^{2}\right) \in \mathscr{E}_{2}$ and $\mathscr{E}_{2}$ is $h$-convex by Proposition 3.3 , we will be able to conclude that $\left(m, p^{2}\right) \notin \mathscr{E}_{2}$ for $m \in$ ]0, $p^{1}\left[\right.$, which implies that $\mu_{1}\left(p^{2}\right)=p^{1}$. Suppose that $\left(p^{1}-1, p^{2}\right) \in \mathscr{E}_{2}$. Then $g\left(p^{1}-1, p^{2}-1\right)=h\left(p^{1}-1, p^{2}-1\right)$, and since $(m, n) \in \mathscr{E}_{1}$ for $\left.m \in\right] 0, p^{1}[$ and $\mathscr{E}_{1}$ is $v$-convex by Proposition 3.3 , we conclude from (51) that $\left(m, p^{2}-1\right) \in \mathscr{E}_{1}$ for $m \in] 0, p^{1}$ [. In particular, $g$ is $h$-linear on $\left[0, p^{1}\right] \times\left\{p^{2}-1\right\}$ and agrees with $h$ at $\left(p^{1}-1, p^{2}-1\right)$ and at $\left(p^{1}, p^{2}-1\right)$, so $g$ agrees with $h$ at $\left(0, p^{2}-1\right)$; therefore, $h$ and $f$ agree at this point, contradicting (49).

The proof that $q^{2}$ satisfies (11) is similar and is left to the reader.

5. Examples. In this section, we illustrate the techniques developed in Section 4 by explicitly solving three concrete examples.

Problem 1. Solve the optimal switching problem on $D=[0,20] \times[0,20]$ with the following payoff function:

$$
\begin{aligned}
& f(u, 0)=f(u, 20)=40 u-2 u^{2}, \quad u \in\{0, \ldots, 20\}, \\
& f(0, v)=f(20, v)=20 v-v^{2}, \quad v \in\{0, \ldots, 20\}
\end{aligned}
$$

[and $f(u, v)=0$ if $1 \leq u \leq 19$ and $1 \leq v \leq 19$ ].

Since $\max f(0, \cdot)=\max f(20, \cdot)=100$, it is clear that the constant function $h(u, v) \equiv 200$ is a bilinear majorant of $f$ with a type 1 contact with $f$, the two nonempty contact sets being $\mathscr{b}_{1}=\{(10,0)\}$ and $\mathscr{b}_{3}=\{(10,20)\}$. By Theorem 4.4, the solution is of type 1. Applying the formulas in (32), we see that

$$
\phi_{1}^{1}(u)=2 u(u+1) \text { and } \phi_{1}^{2}(v)=v(v+1) .
$$

The values of $\phi_{1}^{1}(u)$ and $\phi_{1}^{2}(v), 0 \leq u, v \leq 10$, are tabulated in Table 1 .

Let $\mathscr{D}_{1}, \ldots, \mathscr{D}_{4}$ be the sets defined in Section 4.3. Given the symmetric nature of $f, \phi_{4}^{1}(u)=\phi_{1}^{1}(u)$; therefore, the linear function defined on $\left[0, N^{2}\right]$ (where $N^{2}=20$ ) with value $\phi_{1}^{1}(u)$ at 0 and value $\phi_{4}^{1}(u)$ at $N^{2}$ is constant, equal to $\phi_{1}^{1}(u)$. Since $\phi_{1}^{1}(6)=84<\max f(0, \cdot)=100 \leq 112=\phi_{1}^{1}(7)$, we conclude that inf $\mathscr{D}_{1}=7$ and, by symmetry, sup $\mathscr{D}_{3}=13$. Therefore, Case A of Section 4.3 holds. 
TABLE 1

The functions $\phi_{1}^{1}$ and $\phi_{1}^{2}$ for Problem 1

\begin{tabular}{rrrrrrrrrrrr}
\hline $\boldsymbol{u}$ or $\boldsymbol{v}$ & $\mathbf{0}$ & $\mathbf{1}$ & $\mathbf{2}$ & $\mathbf{3}$ & $\mathbf{4}$ & $\mathbf{5}$ & $\mathbf{6}$ & $\mathbf{7}$ & $\mathbf{8}$ & $\mathbf{9}$ & $\mathbf{1 0}$ \\
\hline$\phi_{1}^{1}(u)$ & 0 & 4 & 12 & 24 & 40 & 60 & 84 & 112 & 144 & 180 & 220 \\
$\phi_{1}^{2}(v)$ & 0 & 2 & 6 & 12 & 20 & 30 & 42 & 56 & 72 & 90 & 110 \\
\hline
\end{tabular}

By Theorem 4.6, $p^{1}=7$ and from (33) and Table 1 we see that $p^{2}=9$, since $\phi_{1}^{2}(8)=72 \leq 84=\phi_{1}^{1}\left(p^{1}-1\right)<90=\phi_{1}^{2}(9)$. By symmetry, $q^{1}=13$ and $q^{2}=11$.

Using Table 1, we now apply Theorem 4.2 to determine $\xi_{1}(v), v=1, \ldots, 8$. On the interval $] p^{2}, N^{2}[$, the analogue of formula (16) is

$$
\xi_{1}\left(x^{2}\right)= \begin{cases}\left.\left.\inf \left\{x^{1} \in\right] 0, p^{1}\right]: \phi_{4}^{1}\left(x^{1}\right) \geq \phi_{4}^{2}\left(x^{2}\right)\right\}, & \text { if }\{\} \neq \varnothing \\ p^{1}, & \text { if }\{\}=\varnothing .\end{cases}
$$

Since $\phi_{4}^{1}\left(x^{1}\right)=\phi_{1}^{1}\left(x^{1}\right)$ and $\phi_{4}^{2}\left(x^{2}\right)=\phi_{1}^{2}\left(20-x^{2}\right)$, we can determine $\xi_{1}(v)$, $v=10, \ldots, 19$. The values of $\xi_{1}(v)$ for $v=0, \ldots, 10$ are shown in Table 2 . The values of $\xi_{1}(v), v=11, \ldots, 19$, are determined by the relation $\xi_{1}(v)=$ $\xi_{1}(20-v)$.

TABLE 2

The function $\xi_{1}$ for Problem 1

\begin{tabular}{cccccccccccc}
\hline $\boldsymbol{v}$ & $\mathbf{0}$ & $\mathbf{1}$ & $\mathbf{2}$ & $\mathbf{3}$ & $\mathbf{4}$ & $\mathbf{5}$ & $\mathbf{6}$ & $\mathbf{7}$ & $\mathbf{8}$ & $\mathbf{9}$ & $\mathbf{1 0}$ \\
\hline$\xi_{1}(v)$ & 0 & 1 & 2 & 2 & 3 & 4 & 5 & 5 & 6 & 7 & 7 \\
\hline
\end{tabular}

Given the symmetry properties of $f$ and Proposition 3.4, this is enough to determine the regions where the vertical and horizontal directions are optimal. These regions are shown in Figure 3. In this figure, we have drawn a short segment through each grid point of the square $] 0,20[\times] 0,20[$ to indicate the optimal direction. If both directions are optimal, two perpendicular segments are drawn. The dots that surround the square provide a "flat representation" of the graph of $f$ restricted to $\partial D$ : the distance from each dot to the nearest point on the square [e.g., $(i, 0)]$ is proportional to the value of $f$ at that point [e.g., $f(i, 0)$ ]. [Note that since $\phi_{1}^{1}(2)=\phi_{1}^{2}(3)$, it is possible to verify that both directions are optimal at $(2,3)$. The same is true for $(18,3),(18,17)$ and $(2,17)$.

Problem 2. Let $b=1$. Solve the optimal switching problem on $D=$ $[0,20] \times[0,20]$ with the following payoff function:

$$
\begin{aligned}
& f(u, 0)=26 u-u^{2}, \quad f(u, 20)=f(20-u, 0), \quad u \in\{1, \ldots, 19\}, \\
& f(20, v)=b\left(26 v-v^{2}\right), \quad f(0, v)=f(20,20-v), \quad v \in\{1, \ldots, 19\},
\end{aligned}
$$




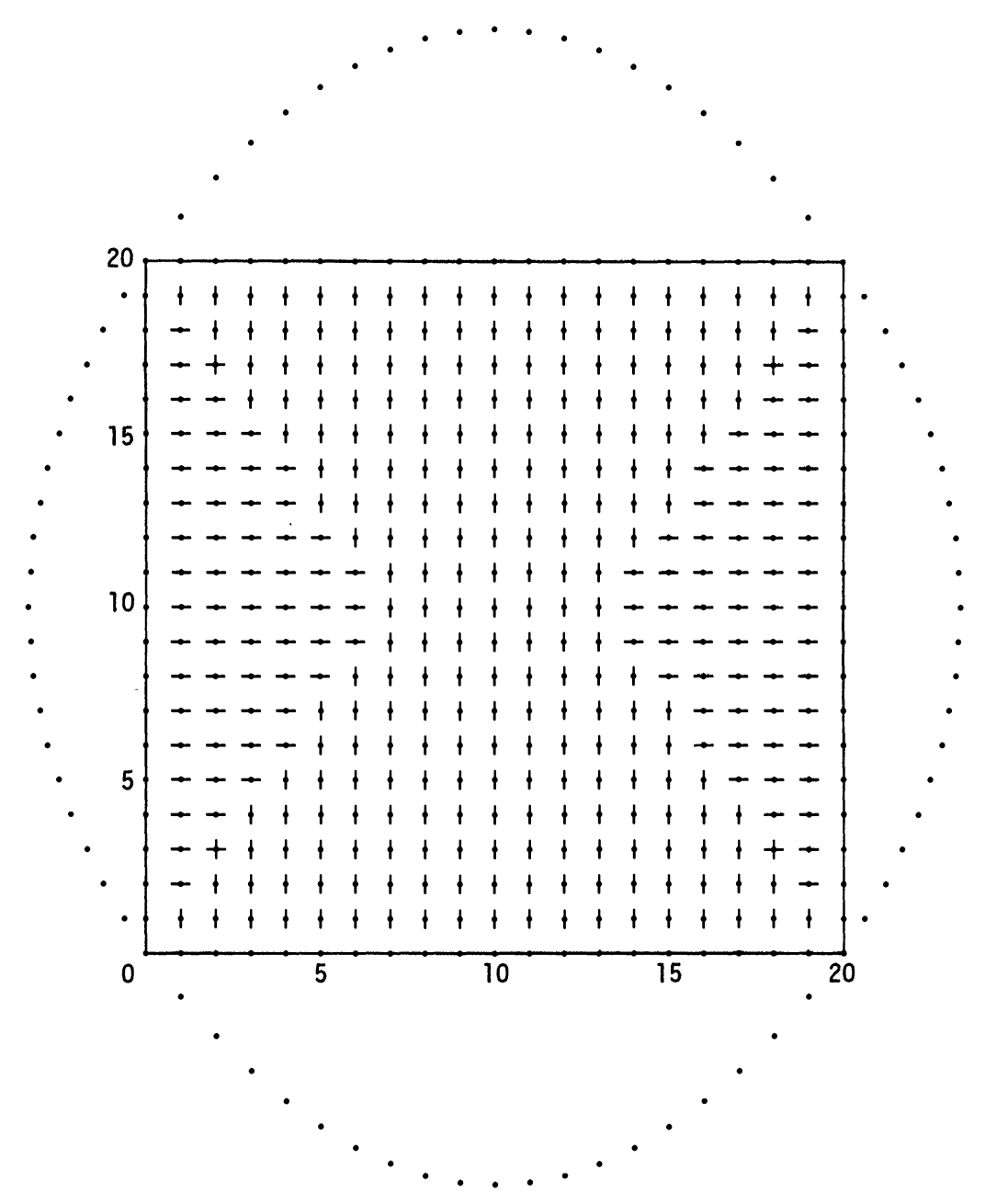

Fig. 3. The solution to Problem 1.

$f(0,0)=f(20,0)=f(20,20)=f(0,20)=0$ and $f(u, v)=0$ if $1 \leq u \leq 19$ and $1 \leq v \leq 19$.

Since $b=1$ and $\sup f(\cdot, 0)=\sup f(20, \cdot)=\sup f(\cdot, 20)=\sup f(0, \cdot)=169$, it is clear that the constant function $h(u, v)=169$ is a bilinear majorant of $f$ with a type 2 contact with $f$, the four contact sets being $b_{1}=\{(13,0)\}$, $\mathscr{C}_{2}=\{(20,13)\}, \mathscr{C}_{3}=\{(7,20)\}$ and $\mathscr{C}_{4}=\{(0,7)\}$. Therefore, the solution is of type 2 by Theorem 4.4 , and by Theorem 4.7 ,

$$
p^{1}=13, \quad q^{1}=7, \quad p^{2}=7 \quad \text { and } \quad q^{2}=13 .
$$


Using (32), the formulas for the functions $\phi_{1}^{1}$ and $\phi_{1}^{2}$ are seen to be $\phi_{1}^{1}(u)=$ $u(u+1)$ and $\phi_{1}^{2}(v)=v^{2}+v+120$. These are easily tabulated. In view of the symmetric nature of $f$, Theorem 4.2 leads to the solution to the problem. We leave the details to the reader, since we prefer to solve a harder problem.

Problem 3. Let $b=0.9$. Solve the optimal switching problem as defined in Problem 2, but with this new value of $b$.

This problem is quite interesting, since it is no longer clear whether the solution is of type 1 or 2 and, in addition, we must determine the fixed point of the transformation $T$ defined in (38). One might expect that determining this fixed point would require some sort of iteration scheme, but in fact it turns out that the solution to Problem 3 can be determined by a finite number of elementary computations.

Using (32) and analogous formulas for $\phi_{i}^{j}$, one finds that

$$
\begin{aligned}
\phi_{1}^{1}(u) & =u(u+1), & \phi_{2}^{1}(u) & =u^{2}-41 u+540 \\
\phi_{2}^{2}(v) & =0.9 v(v+1), & \phi_{3}^{2}(v) & =0.9\left(v^{2}-41 v+540\right) .
\end{aligned}
$$

Given the symmetry properties of $f$, we can now tabulate the eight functions $\phi_{i}^{j}, j=1,2, i=1,2,3,4$. The values of these functions for certain values of $u$ and $v$ are shown in Table 3 .

TABLE 3

The functions $f$ and $\phi_{\imath}^{j}$ for Problem 3

\begin{tabular}{crrrrrr}
\hline $\boldsymbol{u}$ & $\mathbf{8}$ & $\mathbf{9}$ & $\mathbf{1 0}$ & $\mathbf{1 1}$ & $\mathbf{1 2}$ & $\mathbf{1 3}$ \\
\hline$f(u, 0)$ & 144 & 153 & 160 & 165 & 168 & 169 \\
$f(u, 20)$ & 168 & 165 & 160 & 153 & 144 & 133 \\
$\phi_{1}^{1}(u)$ & 72 & 90 & 110 & 132 & 156 & 182 \\
$\phi_{2}^{1}(u)$ & 276 & 252 & 230 & 210 & 192 & 176 \\
$\phi_{3}^{1}(u)$ & 156 & 132 & 110 & 90 & 72 & 56 \\
$\phi_{4}^{1}(u)$ & 192 & 210 & 230 & 252 & 276 & 302 \\
\hline $\boldsymbol{v}$ & $\mathbf{1 1}$ & $\mathbf{1 2}$ & $\mathbf{1 3}$ & $\mathbf{1 4}$ & $\mathbf{1 5}$ & $\mathbf{1 6}$ \\
\hline$f(0, v)$ & 137.7 & 129.6 & 119.7 & 108.0 & 94.5 & 79.2 \\
$f(20, v)$ & 148.5 & 151.2 & 152.1 & 151.2 & 148.5 & 144.0 \\
$\phi_{1}^{2}(v)$ & 226.8 & 248.4 & 271.8 & 297.0 & 324.0 & 352.8 \\
$\phi_{2}^{2}(v)$ & 118.8 & 140.4 & 163.8 & 189.0 & 216.0 & 244.8 \\
$\phi_{3}^{2}(v)$ & 189.0 & 172.8 & 158.4 & 145.8 & 135.0 & 126.0 \\
$\phi_{4}^{2}(v)$ & 81.0 & 64.8 & 50.4 & 37.8 & 27.0 & 18.0 \\
\hline
\end{tabular}


With tedious but elementary computations, one finds that

$$
\inf \mathscr{D}_{1}=11, \quad \sup \mathscr{D}_{3}=9, \quad \inf \mathscr{D}_{2}=12, \quad \sup \mathscr{D}_{4}=8
$$

and so, by Theorem 4.7, the solution to Problem 3 is of type 2 . Let $T$ and $T_{1}, \ldots, T_{4}$ be the transformations defined in (38). Given the symmetry properties of $f$, observe that $T_{3}(\cdot)=T_{1}(\cdot)$ and $T_{4}(\cdot)=T_{2}(\cdot)$.

Let $\alpha_{i}(\cdot)$ and $\beta_{i}(\cdot), i=1, \ldots, 4$, be the functions defined in the proof of Theorem 4.7. By Theorem 4.7, the transformation $T$ has a unique fixed point $x^{3}$ in the interval $[110,402.8]$ [since $\phi_{1}^{1}(10)=110, \phi_{2}^{2}(11)=118.8$ and $\left.T_{1}^{-1}(118.8)=402.8\right]$.

Notice that $T_{1}\left(\phi_{1}^{1}(11)\right)=\phi_{2}^{1}(12)=192$. Since $\phi_{2}^{2}(14)=189<192<216=$ $\phi_{2}^{2}(15), \alpha_{2}(192)=\beta_{2}(192)=15$; so, by a formula analogous to (48),

$$
T_{2}(192)=192+20 \frac{f(20,15)-192}{15}=134
$$

Similarly, $T_{3}(134)=190.67$ (numerical results are rounded to the second decimal) and $T_{4}(190.67)=134.44$. In the same way, we find that

$$
\begin{aligned}
& T_{1}\left(\phi_{1}^{1}(12)\right)=\phi_{2}^{1}(13)=176, \quad T_{2}(176)=140.57, \\
& T_{3}(140.57)=186.28, \quad T_{4}(186.28)=136.17 \text {. }
\end{aligned}
$$

In conclusion,

$$
134.44=T\left(\phi_{1}^{1}(11)\right)>\phi_{1}^{1}(11)=132
$$

and

$$
136.17=T\left(\phi_{1}^{1}(12)\right)<\phi_{1}^{1}(12)=156 .
$$

By Theorem 4.7, $134.44<x^{3}<136.17$.

In view of the tabulated values of $\phi_{i}^{j}$ in Table 3 , we see that $\alpha_{1}(134.44)=12$ and $\beta_{1}(136.17)=12$, from which we conclude that $\alpha_{1}\left(x^{3}\right)=\beta_{1}\left(x^{3}\right)=12$ and, therefore, $p^{1}=\inf \mathscr{C}_{1}=12$ by Theorem 4.7. Note that $T_{1}(134.44)=190.37$ and $T_{1}(136.17)=189.22$. Since $\alpha_{2}(189.22)=\beta_{2}(190.37)=15$, we conclude that $\alpha_{2}\left(T_{1}\left(x^{3}\right)\right)=\beta_{2}\left(T_{1}\left(x^{3}\right)\right)=15$ and, therefore, that $q^{2}=\sup \mathscr{C}_{2}=15$ by Theorem 4.7. The symmetry properties of $f$ imply that $p^{2}=5$ and $q_{1}=8$. Using Theorem 4.2, it is now straightforward to determine the graph of $\xi_{1}$ and to complete the solution of Problem 3. This solution is represented in Figure 4, using the same conventions as in Figure 3.

Acknowledgment. Section 5 was added at the request of an Associate Editor. 


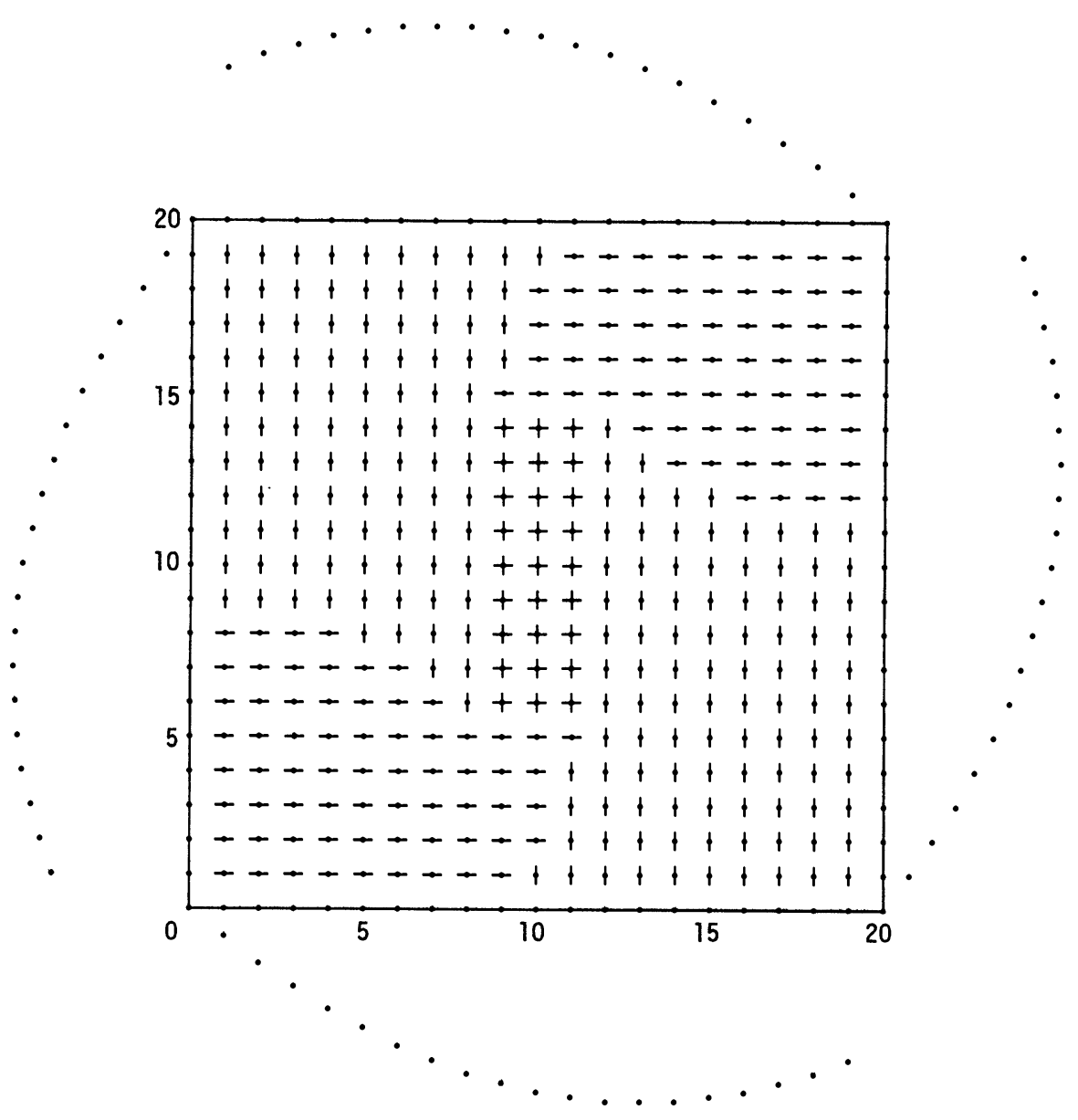

FIG. 4. The solution to Problem 3.

\section{REFERENCES}

[1] CAIroli, R. and DALANG, R. C. (1994). Optimal switching between two Brownian motions. In Stochastic Analysis (M. C. Cranston and M. A. Pinsky, eds.) Proceedings of Symposia in Pure Math. 57 53-63. Amer. Math. Soc., Providence, RI.

[2] Dynkin, E. B. and Yushkevich, A. A. (1969). Markov Processes Theorems and Problems. Plenum, New York.

[3] El KarouI, N. (1981). Les aspects probabilistes du contrôle stochastique. Ecole d'Eté de Probabilités de Saint-Flour. Lecture Notes in Math. 876 74-239. Springer, Berlin.

[4] Evans, L. C. and FrIEDMAN, A. (1979). Optimal stochastic switching and the Dirichlet problem for the Bellman equation. Trans. Amer. Math. Soc. 253 365-389.

[5] Fleming, W. H. and Soner, H. M. (1993). Controlled Markov Processes and Viscosity Solutions. Springer, Berlin.

[6] KRYLOV, V. (1972). Control of a solution of a stochastic differential equation. Theory Probab. Appl. 14 114-130. 
[7] Kushner, H. J. and DupuIs, P. (1992). Numerical Methods for Stochastic Control Problems in Continuous Time. Springer, Berlin.

[8] Mandelbaum, A. (1988). Navigating and stopping multi-parameter processes. In Stochastic Differential Systems, Stochastic Control Theory and Applications. (W. Fleming and P. L. Lions, eds.) 339-372. Springer, Berlin.

[9] Mandelbaum, A., Shepp, L. and Vanderbei, R. J. (1990). Optimal switching between a pair of Brownian motions. Ann. Probab. 18 1010-1033.

[10] Mandelbaum, A. and VANDERBei, R. J. (1981). Optimal stopping and supermartingales over partially ordered sets. Z. Wahrsch. Verw. Gebiete. 57 253-264.

[11] VANDERBEI, R. J. (1992). Optimal switching among several Brownian motions. SIAM J. Control Optim. 30 1150-1162.

DÉPARTEMENT DE MATHÉMATIQUES

ECOle Polytechnique FÉdÉRALE

1015 LAUSANNE

SWITZERLAND
DEPARTMENT OF MATHEMATICS

TUFTS UNIVERSITY

MEDFORD, MASSACHUSETTS 02155 